\title{
A data-driven perspective on the hierarchical assembly of molecular structures
}

\author{
Lorenzo Boninsegna, ${ }^{\dagger}$ Ralf Banisch, ${ }^{\ddagger}$ and Cecilia Clementi ${ }^{*, \dagger}, \ddagger$ \\ $\dagger$ Department of Chemistry, and Center for Theoretical Biological Physics, Rice University, \\ 6100 Main St, Houston TX 77005, USA \\ $\ddagger$ Department of Mathematics and Computer Science, Freie Universität Berlin, Arnimallee \\ 6, 14195 Berlin, Germany \\ E-mail: cecilia@rice.edu
}

\begin{abstract}
Macromolecular systems are composed of a very large number of atomic degrees of freedom. There is strong evidence suggesting that structural changes occurring in large biomolecular systems at long timescale dynamics may be captured by models coarser than atomistic, although a suitable or optimal coarse-graining is a priori unknown. Here we propose a systematic approach to learning a coarse representation of a macromolecule from microscopic simulation data. In particular, the definition of effective coarse variables is achieved by partitioning the degrees of freedom both in the structural (physical) space, and in the conformational space. The identification of groups of microscopic particles forming dynamical coherent states in different metastable states leads to a multiscale description of the system, in space and time. The application of this approach to the folding dynamics of two proteins provides a revised view of the classical idea of pre-structured regions (foldons) that combine during a protein folding process, and suggests a hierarchical characterization of the assembly process of folded structures.
\end{abstract}

\section{Introduction}

The last several years have seen an immense increase in high-throughput and high- performance techniques to simulate molecular systems at a microscopic level, ${ }^{1-3}$ that has, in turn, stimulated the surge of powerful data analysis techniques to extract essential features, collective variables or representative states from simulations ${ }^{4-6}$ in order to reconcile them with experimental data. These new techniques have provided tremendous help in advancing our understanding of macromolecular processes (e.g., see refs. 7,8). However, even if the simulation of considerably-sized molecular systems over milliseconds is now feasible, the same approach is not possible for large macromolecular complexes, thus leaving a gap when attempting to scale to cellular signaling.

Empirical and theoretical results indicate that for most macromolecular processes only a limited fraction of the phase space is relevant, and most of the dynamics is not "interesting" as it consists of fast and local fluctuations around long-lived (metastable) conformational states. ${ }^{9-12}$ In contrast, the rarely observed transitions between metastable states are crucial, as they govern the switching between biologically relevant functions. Recent work ${ }^{13,14}$ has shown that, for medium/long time scales (generally on the range $\gtrsim 10 \mathrm{~ns}$ ), a very small number of parameters are enough to describe the coarse dynamics of a large macromolecular system. These results suggest that it may be possible to reproduce the thermodynamics and long timescale kinetics of a macromolecu- 
lar system by means of reduced models, using a significantly smaller number of degrees of freedom. Simulation of coarse models may have a significantly reduced computational burden, and allow the study of larger systems on longer timescales. ${ }^{15-17}$ Moreover, by filtering out nonessential details coarse models allow a more direct identification of the essential physical ingredients needed to reproduce a macromolecular process, a key step towards the formulation of the "rules" regulating the behavior of biomolecular processes at different scales.

However, there is still, as yet, no general solution on how to select an optimal set of effective degrees of freedom to reproduce the long timescale dynamics of a given system. The choice of the coarse coordinates is usually made by replacing a group of atoms by one effective particle, usually based on physical and chemical intuition. Because of the local geometric regularity of a protein backbone or a DNA structure, popular models reduce the complexity of a macromolecule to a few interaction sites per residue or nucleotide, e.g., the $C_{\alpha}$ and $C_{\beta}$ atoms for a protein. ${ }^{18-22}$

Complementary mathematical work has suggested how to define optimal collective variables of macromolecular systems, that is, descriptors that can identify interesting collective phenomena over long timescales, and separate macroscopically different structures or aggregation states. ${ }^{5,6}$ In a sense, the description of the macromolecular dynamics in terms of very few descriptors can be considered as a form of extreme coarse-graining where the thermodynamics and kinetics of the slowest processes are well described by a few variables. However, such a mathematically rigorous definition does not readily provide a physically meaningful coarsegrained representation of a macromolecular system in terms of clusters of atoms, or collections of internal coordinates (angles, distances). In practice, one would like to use a coarse representation of the system that is both physically interpretable and satisfies some criterion of optimality in reproducing the microscopic dynamics.

We propose here to use mathematical ideas similar to what has been developed to identify slow variables ${ }^{6}$ but extend the analysis to include the chemical structure of the molecule. The definition of collective variables associated with the slow processes allows to simplify the complex geometry of the high dimensional configurational space in terms of a few metastable regions. In the present work, the geometry we want to represent in minimal form is not only the high dimensional configurational space but includes the molecular structure itself: we combine the partitioning of the overall configurational space visited by the molecule with the partitioning of the 3-dimensional structure of the molecule into groups of atoms that display dynamical coherence.

The minimal representation of the system dynamics as a network of few metastable states, their relative population, and the transition rates between them is usually referred to as a Markov State Model (MSM). ${ }^{4,11,23-25}$ For each metastable state of an MSM, we seek an optimal coarse-graining of the molecular structure in terms of groups of atoms that can be considered effective dynamical "blocks". The resulting groups of atoms preserve some structural integrity during the system dynamics, and at the same time capture the slow processes of the system. We name this approach as the Structure and State Space Decomposition $\left(\mathrm{S}^{3} \mathrm{D}\right)$. We show that $\mathrm{S}^{3} \mathrm{D}$ provides different coarse grained representations of a molecular structure in different metastable regions of the system: the optimal resolution to describe the system dynamics changes as a macromolecule visits different metastable states.

We illustrate $\mathrm{S}^{3} \mathrm{D}$ by applying it to molecular simulation trajectories of two different protein systems over $m s$ timescales (generated on the Anton supercomputer $\left.{ }^{2,26}\right)$. The results illustrate the mechanism of transitions between protein models at different resolution associated with different macroscopic states of the system. The formation and disassembly of different groups of atoms into coherent domains in different metastable states presents a multiscale characterization of the system dynamics, both in conformation space and chemical structure.

Interestingly, the results provide a quantification and new interpretation (in terms of coher- 
ent dynamical structures) of the idea of modular units in proteins (foldons), ${ }^{27}$ and their hierarchical formation and assembly. ${ }^{28}$ We show that, while groups of atoms forming constitutive blocks can be clearly detected in the dynamics, these groups are highly heterogeneous in size and composition, and not always associated with the formation of secondary structure. The comparison of the $\mathrm{S}^{3} \mathrm{D}$ results with what is obtained by using the thermodynamic definition of foldons, and the consequences for coarse-graining methods are discussed.

\section{Results and discussion}

\section{Dynamical coherent groups of atoms in} a macromolecule We turn to recent results in dynamical system theory to define a general and robust approach to identify a minimal number of dynamically coherent domains in proteins or protein complexes, and obtain a faithful description of macromolecular conformational rearrangements over long timescales in a reductionist fashion.

The notion of coherence has attracted considerable attention in the mathematics community in the recent past. ${ }^{31,32}$ As a typical example, consider a number of particles released in close proximity to each other. The goal is to identify the particles that will remain mutually close to each other for some time. For instance, when caught in the same current stream, drifters released in the ocean will move together as a group even if the current carries them around the globe. These groups of particles form coherent sets. ${ }^{32}$ Here we demonstrate that this idea can also be used in the context of a macromolecular system, where the role of the ocean drifters is played by the individual atoms, and we want to determine the groups of atoms that move coherently. In order to preserve the long timescale processes of a macromolecular system, we combine the partitioning of the coherent groups of atoms in a molecular structure with the partitioning of the conformation space (by means of a Markov State Model analysis ${ }^{4}$.

The Diffusion Map approach ${ }^{33}$ has been shown to provide a clear geometric inter- pretation $^{14}$ in the dimensionality reduction of high dimensional systems, and to obtain low dimensional energy landscapes of macromolecules. ${ }^{12,29,34-37}$ In order to solve the problem of finding coherent sets of atoms from molecular dynamics trajectories, we employ a version of Diffusion Map extended to the time dimension ("time-averaged Diffusion Map") that has been recently proposed in the context of dynamical systems. ${ }^{31}$ In a nutshell, the Diffusion Map approach applied to the atomic positions in a single molecular configuration constructs a Markov probability matrix based on which pairs of atoms are close to each other. The dominant eigenfunctions of this Markov matrix then capture the geometry of the given molecular configuration. The time-averaged Diffusion Map considers an ensemble of configurations generated by molecular dynamics and constructs a Markov probability matrix based on which pairs of atoms stay close to each other on average over all configurations in the ensemble. The ensemble we consider here consists of all configurations that are in a certain partition of the conformational space (metastable state), as identified by a Markov State Model analysis. The configurations could be sampled either from one long or multiple short trajectories. The eigenfunctions of the time-averaged Diffusion Map Markov matrix are dynamical coordinates, and geometric clustering in their space returns groups of atoms which are mutually close over the whole time range considered, and are therefore coherent. Details on the time-averaged Diffusion Map method and its implementation are provided in the Supporting Information.

We apply this approach to the subset of molecular dynamics trajectories within each metastable state, as found by a Markov model analysis, resulting in the definition of a strategy for the Structure and State Space Decomposition $\left(\mathrm{S}^{3} \mathrm{D}\right)$ of the macromolecular dynamics.

The identification of coherent structural "domains" in different regions of the conformational landscape of a macromolecule allows us to identify the minimal structural "units" that remain coherent in every region of the landscape, and illustrate the assembly or disassem- 

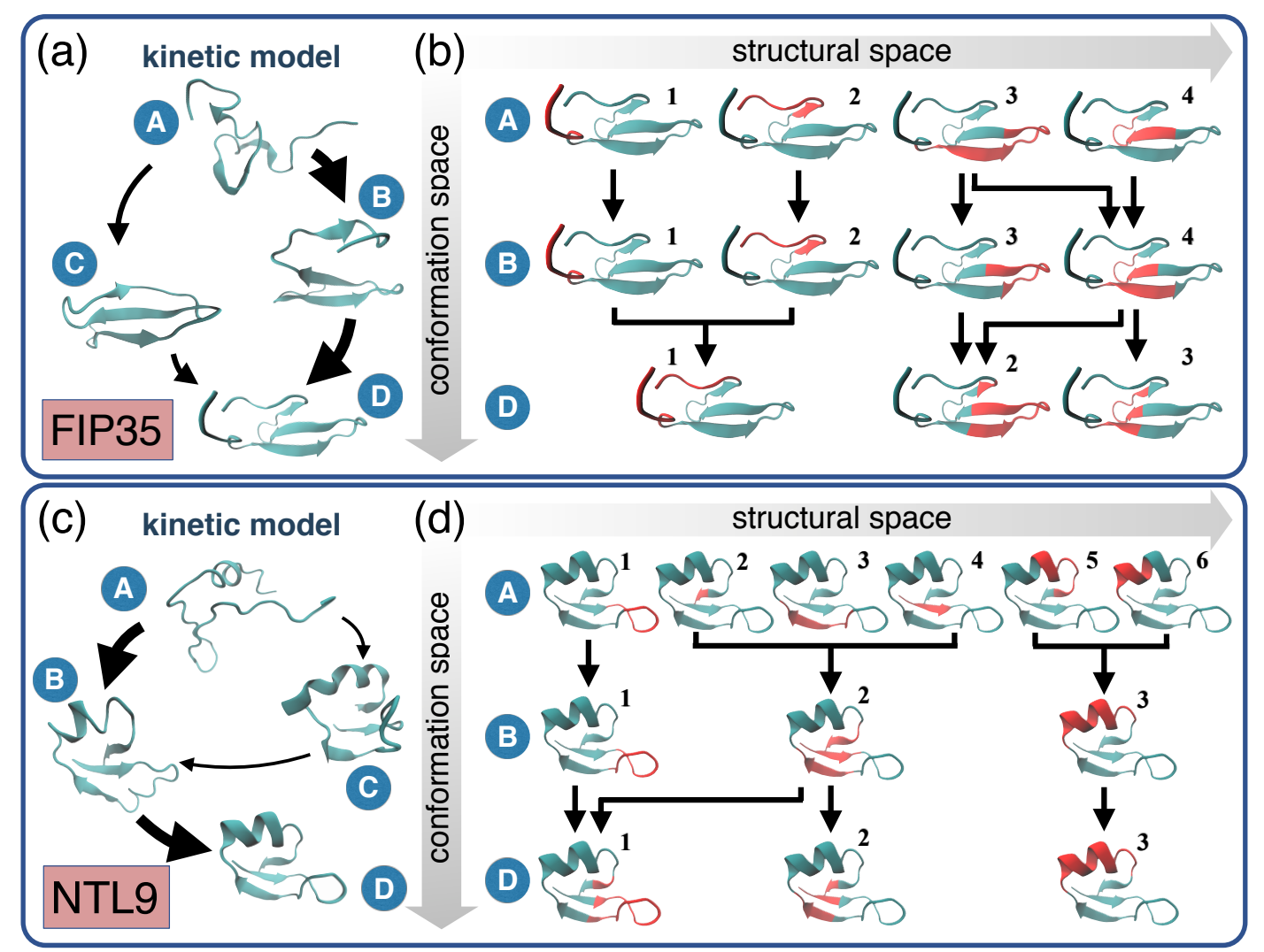

Figure 1: Kinetic network and coherent set analysis results obtained by $\mathrm{S}^{3} \mathrm{D}$ for protein FIP35 (a-b) and NTL9 (c-d). (a) and (c): Schematic representation of the kinetic models; transition fluxes between states are indicated with arrows of widths proportional to the intensity of transition probabilities. FIP35 (a) folds through the sequence $A \rightarrow B \rightarrow D$ or $A \rightarrow C \rightarrow D$, where state $A$ is the unfolded state, $D$ is the folded state, and $B$ and $D$ are two different intermediate states. Protein NTL9 (c) folds along the sequence $A \rightarrow B \rightarrow D$ or $A \rightarrow C \rightarrow B \rightarrow D$. State $A$ is the unfolded state, $D$ is the folded state, $B$ is an on-pathway intermediate, and $C$ is a misfolded intermediate (not kinetically connected to the folded state). For both proteins, the first pathway $(A \rightarrow B \rightarrow D)$ dominates the folding dynamics as its transition flux is about three orders of magnitude larger than the other pathway. These kinetics models are consistent with previous studies. ${ }^{29,30}$ (b) and (d): Results from the time-averaged Diffusion Map analysis along the most likely folding pathways. The different structural coherent domains labeled with numbers and highlighted in red on the folded structure in a each metastable state are the coherent sets identified by $\mathrm{S}^{3} \mathrm{D}$. different metastable states. Black arrows indicate the changes in the structural clusters as a molecule transitions between the different metastable states. Results for the less likely folding pathways are reported in the Supplementary Information. 
bly of these units to form the different functional states. In the following we describe the results from the application of $S^{3} \mathrm{D}$ to obtain a minimal representation (both in configuration and structure space) of the folding dynamics of two different proteins for which long equilibrium all-atom trajectories trajectories are available. ${ }^{2,26}$

Details on the implementation of the Markov Model, the time-averaged Diffusion Map, the spectral clustering, the choice of parameters, and the validation are provided in the Supplementary Information.

\section{Coarse-grained kinetic models of proteins}

We use $S^{3} D$ to discover the hierarchical dynamical processes in the folding of two proteins, for which long equilibrium all-atom trajectories trajectories are available: FIP35 WW-domain, ${ }^{2}$ and NTL9. ${ }^{26}$

The resulting coarse-grained kinetic model is illustrated in Fig. 1, both for FIP35 (ab) and NTL9 (c-d). Figs. 1(a) and (c) show that, for both proteins, the folding dynamics mainly proceeds through two possible pathways. Figs. 1(b) and (d) illustrate the decomposition of the structures in each state into coherent domains (red), and how these domains change along the most likely folding pathway (see Supporting Information for the less probably pathways). The arrows connecting coherent domains in different metastable states indicate if such domains preserve their identity, split into separate ones, or merge together as the proteins proceed along the primary folding pathways. In the following, we indicate as $X i$ the $i^{\text {th }}$ cluster in metastable state $X \in\{A, B, C, D\}$, with $i \in\left\{1, \ldots, n_{X}\right\}$, where $n_{X}$ is the total number of coherent domains for state $X$.

The dynamics of the coherent domains in FIP35 (Fig. 1b) shows that the $\mathrm{N}$ and $\mathrm{C}$ terminal tails of the proteins move independently in the unfolded (clusters $A 1$ and $A 2$ ) and intermediate state ( $B 1$ and $B 2$ ), and merge into a single coherent domain $(D 1)$ in the folded state. In the FIP35 protein core, two separate clusters are detected in the unfolded state $(A 3$ and $A 4)$ which undergo a "domain exchange" process during folding: a piece of $A 3$ corresponding to a hairpin segment detaches and is absorbed by $A 4$ to generate coherent domains $B 3$ and $B 4$ in the intermediate state, that, in turn, split unevenly into domains $D 2$ and $D 3$ in the folded state. In essence, the major folding pathway of FIP35 consists in the splitting and merging of clusters $A 3$ and $A 4$, and the assembly of clusters $A 1$ and $A 2$. A similar scenario occurs in the major folding pathway of the NTL9 protein. The transition from the unfolded state to the intermediate corresponds to the assembly of different coherent clusters into larger coherent domains: $A 2+A 3+A 4$ merge into $B 2$, and $A 5+A 6$ into $B 3$, while $A 1$ remains an independent domain. The transition from the intermediate to the folded state involves the splitting and merging of coherent domains $B 1+B 2$ into $C 1+C 2$, while the previously assembled alpha helical domain $B 3$ is maintained.

Not surprisingly, for both proteins the unfolded state is decomposed into a larger number of coherent domains than the intermediate or folded states. Coherent domains in the unfolded states always correspond to connected regions along the protein sequence indicating that, although the proteins appear partitioned into stretches of sequence that move coherently, the different stretches move independently from each other, as expected in the absence of collective motions. On the other hand, coherent domains in the intermediate and native states comprise disconnected regions (i.e. groups of atoms far apart along the sequence are assigned to the same coherent domain), signaling the presence of tertiary structure and long-range order.

Fig. 2 confirms that these coherent domains capture most of the tertiary structure of a protein in a given metastable state. The probability of contact formation in state $A, B, C$, and $D$ are shown for protein FIP35 (left) and NTL9 (right). In each metastable state, the colored areas on the upper contact matrix indicate all the contacts that can be formed by pairs of residues inside a given coherent domain, and different colors correspond to different structural domains. In each metastable state, all contacts with non-negligible probability of formation essentially fall inside a colored region of 
the contact space in Fig. 2. That is, each domain captures a set of contacts occurring in the metastable state (either native or non-native), and almost no contact is formed between atoms belonging to separate coherent domains. These results offer a complementary view to what is presented in Fig. 1 and Fig. S6. In both proteins, metastable state $C$ presents a larger number of non-native contacts than the other states. The comparison between the contact maps of state $C$ and the folded state $D$ shows that the shift in the coherent domain boundaries from $C$ to $D$ corresponds to a rearrangement from out of register to native structural packing.

Although the coherent domains capture most of the secondary and tertiary structure formed in the different metastable states, the structural content is not distributed equally and the domain partitioning can not be easily inferred from the contact maps alone: some coherent domains contain only a marginal number of contacts, whereas others capture a massive number of contacts. This result suggests that the coherent clusters are amenable to a hierarchical interpretation, very similar to the interpretation of the Diffusion Coordinates in standard Diffusion Map. ${ }^{12,14}$ In the latter, Diffusion Coordinates of higher order resolve dynamical details at a finer time resolution and clustering in this coordinate space may return states with different level of metastability. Similarly, in the present context, dynamical coordinates of higher order encode higher "coherence resolution", and the structural coherent domains obtained by clustering in this space are expected to have different level of coherence.

\section{Minimal assembly units as dynamic} building blocks In order to investigate the variations in the coherent domain decomposition in the different metastable states, and how the different domains split and merge in the transition between metastable states, we define the minimal assembly units, $\left\{\mathcal{U}_{i}\right\}$ as the smallest set of complete and disjoint structural units that can be composed to form any coherent domain in any state: $\mathcal{U}_{i} \cap \mathcal{U}_{j}=\emptyset$ and $\bigcup_{i} \mathcal{U}_{i}=\mathcal{P}$, where $\mathcal{P}$ represents the whole protein, and every coherent domain $X i=\bigcup_{j \in J} U_{j}$ for some index set $J$.

As these units never split into subcomponents across all the metastable states, they can be considered the elementary building blocks of the protein, which can assemble in different ways to form different structural ensembles in different regions of the energy landscape. A synoptic representation of the coherent structural domains in terms of their minimal assembly units is given for both proteins and all states in Fig. 3. The decomposition of each state $(A, B, C$, and $D)$ into its coherent domains and the composition of each domain in terms of minimal assembly units is illustrated by the schematics for FIP35 (Fig. 3b), and for NTL9 (Fig. 3f). Coherent domains are shown linearly along the protein sequence, with minimal units indicated as differently-colored stripes, along with the protein sequence and location of the secondary structure. The positions of the minimal units on the native structure is shown in Fig. 3c (FIP35) and Fig. 3g (NTL9) (same colors as in Fig. 3b (FIP35) and Fig. 3f (NTL9)).

Overall, protein FIP35 consists of 13 minimal units, and NTL9 of 14. It is clear from Fig. 3 that the units have very nonuniform sizes. In protein NTL9, one unit comprises a single peptide plane (between ILE4 and PHE5), and several units in both proteins are defined by a single residue (PRO6, ARG11, TYR19, TYR20, SER28, PHE30 in FIP35, and LEU6, ILE27 in NTL9), or even a single side-chain (LYS10 and GLN29 in FIP35, and PHE5 and ILE27 in NTL9). On the other hand, in both proteins there are units encompassing multiple consecutive residues (up to 7 ). The detailed atomic composition of each unit is provided in the Supplementary Information.

Interestingly, the larger units do not necessarily correspond to the location of secondary structure in the proteins. While the structural coherent domains in the native state include the whole helices and most of the strands, assembly units smaller than the full secondary structure elements need to be considered in order to accommodate the coherent domains formed in different metastable states. For instance, the largest domain in the native structure of FIP35 

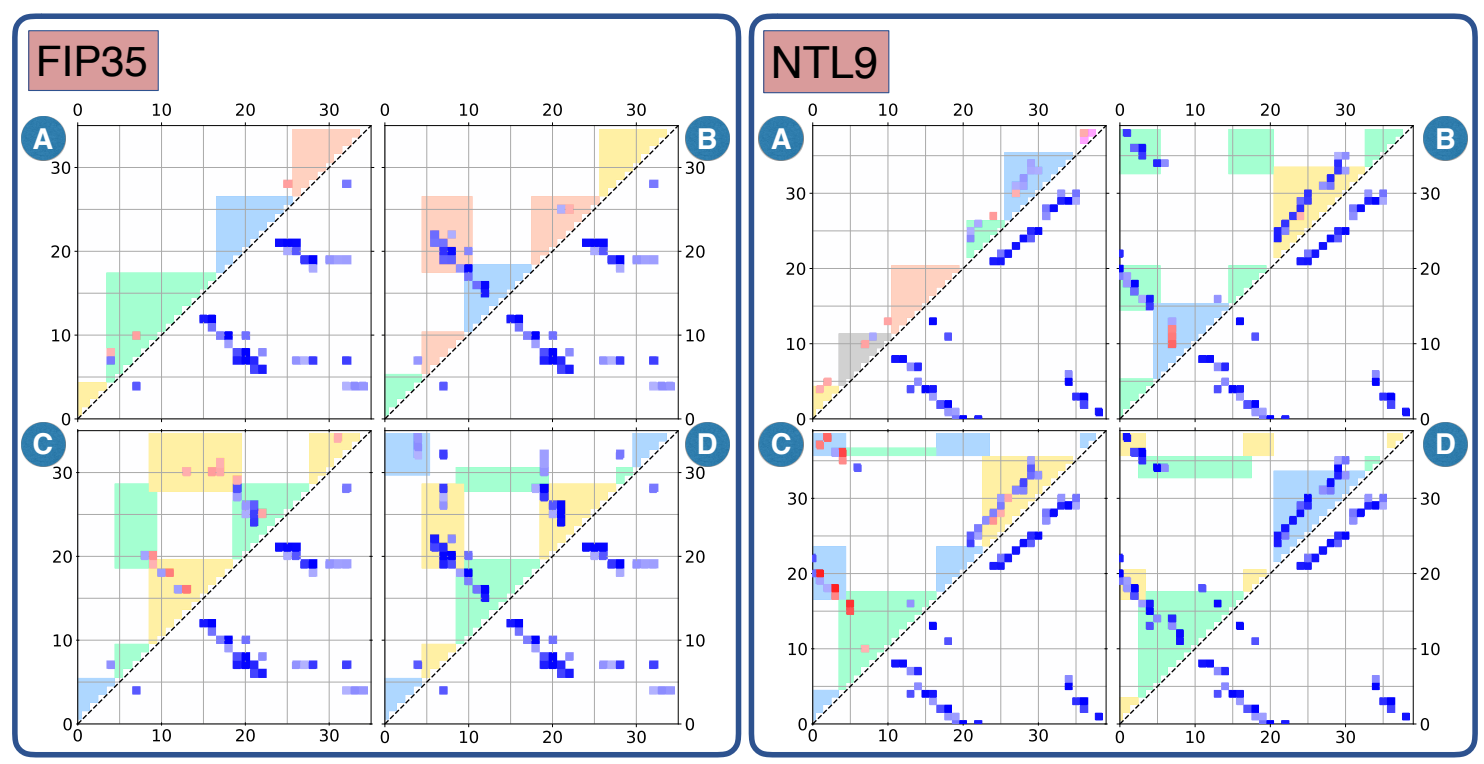

Figure 2: Contact probability versus coherent domains in each metastable state of protein FIP35 (left) and NTL9 (right). Labels $(A, B, C, D)$ identify the states as in Fig. 1. Areas shaded in different color indicate different coherent domains in a metastable state. The color correspondence between different panels is arbitrary: colors are used to distinguish the different coherent domains in each metastable state independently. Native and non-native contacts are shown in blue and red, respectively. The color intensity indicates the value of the contact probability, from white to dark blue or red. In each panel, the lower triangular matrix always shows the contact map of the folded state of the corresponding protein, as a comparison. A residue-residue contact is considered formed if the shortest distance among all pairs of heavy atoms is shorter than a threshold value of $0.35 \mathrm{~nm}$. The contact probability is estimated as the frequency of the contact in a given metastable state. For clarity, only contacts with probability higher than 0.3 are shown. The contact maps of the different metastable states are consistent with what found in previous studies, both for FIP $35^{29}$ and NTL9 ${ }^{38}$. 

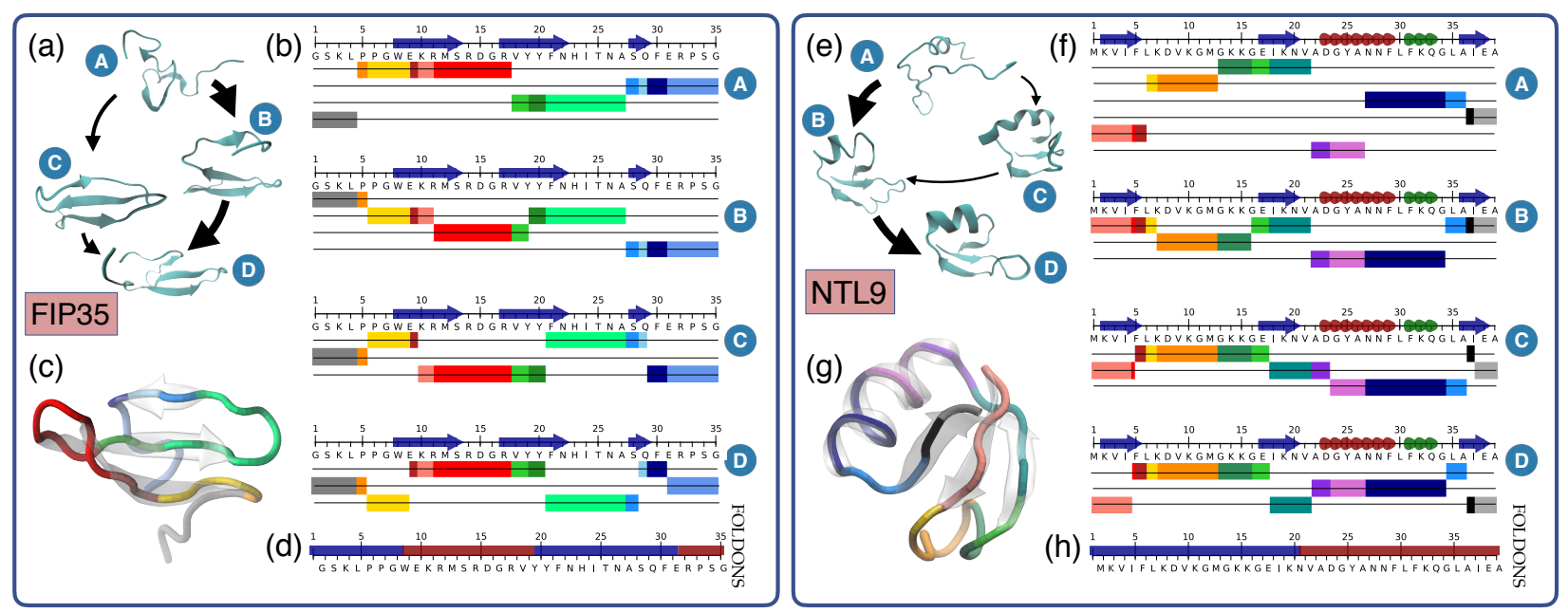

Figure 3: Minimal assembly units for FIP35 (a-c) and NTL9 (e-g) proteins. The same kinetic network models as in Fig. 1 are reported in (a) and (e), as a reference. (b) and (f): The minimal assembly units are illustrated for each protein: different colors are used to distinguish between the different units inside a given state, and the same color is used to identify the same unit across different states. In each metastable state (A, B, C, D, as in Fig. 1), the units are assembled in the corresponding coherent domains along the protein primary sequences. Thin black horizontal lines indicate the different domains in each metastable state, along which the units are placed in a notes-on-the-staff fashion. The locations of native secondary structure on the protein is also reported on the sketch of the protein sequence on top of the coherent domain representation for every state. An $\alpha$ or $3_{10}$ helix is marked by dark red or green chain dots, and a strand by a dark blue arrow. The secondary structure assignment has been obtained by (c) and (g): The same color palettes as in (b) and (f) are used to highlight the minimal units on the protein native structures. As a comparison, the results from a foldon analysis on the two proteins are reported in (d) and (h). Four foldons are identified in protein FIP35 (d) and two in NTL9 (h). The regions corresponding to the different foldons are marked by alternating dark blue and red colors. 
(domain D2 in Fig. 1(b), and the corresponding area shaded in green in state $D$ in Fig. 2) encompasses the bulk of the protein $\beta$-sheet. However, this coherent domain is composed of 7 different minimal units, that can assemble in different ways to form different coherent domains in other metastable states. A similar picture appears for NTL9, where a large coherent domain in the native state (domain D3 in Fig. 1(b), corresponding to the blue area in state $D$ in Fig. 2) contains both the $\alpha$-helix and the consecutive $33_{10}$-helix, but it decomposes in 3 different minimal units that combine to form other coherent domains in other metastable states.

The existence of "building blocks" in proteins has been previously proposed multiple times and explored with different approaches (see, e.g. refs 39-42). Previous studies, however, have mostly focused on energetic or structural factors. Most of existing methods for decomposing proteins into domains are based on the analysis of fluctuations of inter-atomic distances as indicators of rigidity of different parts of the macromolecule. Coordinate fluctuations are usually computed over short trajectories or sets of homologous structures, or by normal mode-analysis. Previous approaches have not included temporal information, or the fact that multiple metastable states can be visited during the protein dynamics. It is well known that structural similarity does not necessarily correlate with kinetic similarity; ${ }^{29,43,44}$ therefore, domains based solely on structural considerations may not capture important dynamical information. Additionally, two-point correlations have been shown to encode a partial correlation content only. ${ }^{45}$

In contrast, $S^{3} \mathrm{D}$ provides a coherence-based domain decomposition by combining the notions of structural and kinetic similarity. Although $\mathrm{S}^{3} \mathrm{D}$ shares some resemblance with parts of previously proposed methods, there are significant differences in the formulation and implementation. In particular, the use of the Diffusion Map construction ${ }^{33}$ to build the similarity matrix allows us to capture nonlinear effects, and is supported by mathematical theory, ${ }^{31,32}$ establishing a rigorous link with the concept of coherence in dynamical systems, and providing an optimality criterion that is missing from other more heuristic formulations. Additionally, the combination of structural and space state analysis of $\mathrm{S}^{3} \mathrm{D}$ allows a state-dependent definition of coherent domain, each of them adequately describing subsets of the configuration space. This is very close in spirit to the idea of "Ultra Coarse-Graining", 46 that argues that state dependent coarse graining variables can provide a more suitable protein model and may improve the performance and the interpretation of the results. Indeed, the final output of $\mathrm{S}^{3} \mathrm{D}$ is a kinetic network where each node represents a metastable state-specific protein decomposition into coherent domains, which is amenable to a dynamic interpretation: the conformational transition from one state to the other is here translated in a step-wise assembly or disassembly of the minimal units.

Foldons revisited The existence of kinetically competent, quasi-independently folding units of a protein was first suggested on the base of geometrical considerations, ${ }^{47}$ tested by means of an energy landscape analysis, ${ }^{27}$ and appears in agreement with experimental results on some proteins. ${ }^{48,49}$ The idea of a hierarchical dynamics was also implemented as a practical method ("zip and assembly") to speed up protein folding simulation. ${ }^{28}$

So called "foldons" have been identified on a number of proteins by considering different protein segments, $\{j\}$ (of length $N_{j}$ ), and estimating the ratio, $\theta_{j}=\frac{\Delta E_{j}}{\delta E_{j} \sqrt{N_{j}}}$, between the energy stability gap, $\Delta E_{j}$, of segment $j$ in the folded configuration (with respect to misfolded alternatives), and its energy variance in the misfolded state(s), $\delta E_{j}$. The energy landscape theory of protein folding ${ }^{50}$ relates this quantity $\theta_{j}$ to the ratio between the folding temperature and the glass transition temperature, therefore providing a measure of relative foldability of a protein segment. By invoking the minimal frustration principle, ${ }^{50}$ it was proposed ${ }^{27}$ that contiguous protein regions that maximize $\theta_{j}$ could be considered fundamental units of protein folding. 
We apply this idea to the two proteins considered here (see Supplementary Information for details). The results are reported in Fig. 3(d) and (h) and show that foldons include the secondary structure in both proteins, and provide a coarser structural decomposition than $S^{3} D$. Interestingly, foldons identified by a purely thermodynamic criterion correlate with the largest assembly units identified by $\mathrm{S}^{3} \mathrm{D}$ on the basis of dynamic considerations. This result is not entirely surprising in the light of the energy landscape theory, ${ }^{50}$ yet it provides an independent validation for $\mathrm{S}^{3} \mathrm{D}$, that is a datadriven approach. Additionally, it is worth noting that the foldons are identified here by using an all-atom force-field to evaluate the energy of the protein fragments in the different states, while the approach was proposed and previously used only with coarse-grained energy models that may significantly smooth out the energetic roughness of a protein folding landscape.

The minimal units shown in Fig. 3(b) and (f) complement and revisit the foldons idea, by providing a finer discretization of the protein structure and adding a dynamical interpretation to the foldability criterion.

\section{Conclusions}

We propose $S^{3} \mathrm{D}$, a rigorous approach to identify dynamically coherent structural domains in macromolecules; i.e. groups of molecular components which move collectively and maintain their identity during the system dynamics. By partitioning both in structural and state space, $\mathrm{S}^{3} \mathrm{D}$ extends to the physical space the idea of data-driven coarse-graining that has been proposed and used in the macromolecular conformation space for the definition of optimal reaction coordinates. In principle, $\mathrm{S}^{3} \mathrm{D}$ can also be tuned to operate on spaces different from that of the atomic Cartesian coordinates used here; e.g. the space of contacts, or other physical observables, which could provide alternative points of view of the dynamics.

The $S^{3} \mathrm{D}$ analysis of the folding mechanism of two proteins, FIP35 and NTL9, show that, although different coherent domains are formed in different metastable regions along the folding process, minimal assembly units can be identified. These structural units can be considered building blocks of the macromolecular dynamics, as all the relevant regions of the molecule state space are formed by their assembly and disassembly. As such, $\mathrm{S}^{3} \mathrm{D}$ provides a truly multiscale characterization of the system dynamics both in conformational space and physical space. Interestingly, this rigorous analysis also revisits the classic idea of foldons, as "maximally foldable" segments that assemble during a protein folding process. ${ }^{27}$

While we have illustrated $\mathrm{S}^{3} \mathrm{D}$ on a protein folding application, it can be used in general to learn the minimal dynamical units in large conformational changes in macromolecules, or in the assembly of super-molecular complexes. As such, it can offer a link between different resolutions, for a systematic upscaling of biophysical models.
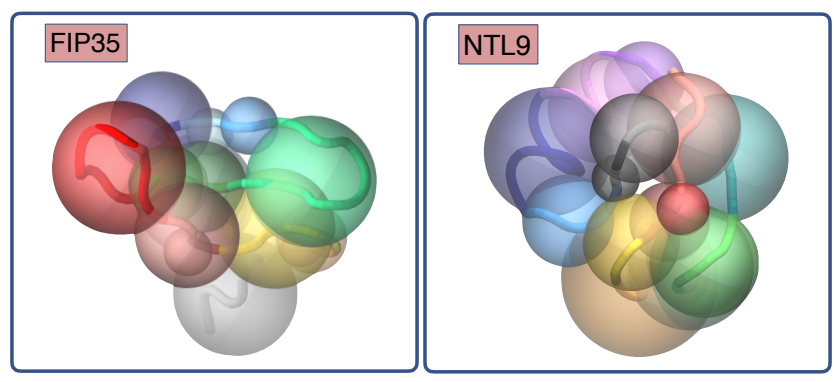

Figure 4: Beads of different colors identify the coarse-grained units for FIP35 (left) and NTL9 (right). The units are defined as the minimal set of structural components that can be composed to form all the different coherent domains in all metastable states of a protein. The backbone of the folded structure is shown in the background as a reference.

The picture emerging from the $\mathrm{S}^{3} \mathrm{D}$ analysis suggests that a global coarse representation of a macromolecule may be inadequate, and different minimalist models should be considered as different metastable states are visited, as it has been advocated in. ${ }^{46}$ A logical consequence is that the minimal assembly units identified here could provide natural candidates for a statedependent coarse-graining approach: the col- 
lections of atoms that preserve their geometric integrity as a function of time across all the relevant states visited by the molecule could be considered as effective "beads" in a coarse-grained model, as pictorially presented in Fig. 4. Such a data-driven coarse-graining could overcome limitations which affect most structural coarse grained models where the choice of the collective degrees of freedom is mostly guided by intuition.

At the level presented here, the results are system-dependent. The coarse-grained representation shown in Fig. 4 is not immediately transferable to different molecules. Additional investigation on common features across a broad range of systems is needed to draw general conclusions and to understand if and what parts of the minimal assembly units could be transferable.

\section{Materials and Methods}

Identification of metastable states The conformational space explored by molecular dynamics trajectories was reduced to a set of metastable states using a Markov State Model (MSM) approach. ${ }^{4}$ All calculations were performed by using implementations available in the pyEMMA software package (pyemma.org). ${ }^{51}$ Details on the implementation, the choice of the model parameters, and validation of the models are provided in the Supplementary Information.

\section{Clustering into coherent groups of atoms}

In each of the metastable states, coherent structural domains were identified by performing agglomerative clustering on the first several dominant eigenvectors. Validation of the clustering was performed by using both the silhouette scoring and the distribution of distance thresholds returned by the agglomerative clustering, and provided a very clear and robust criterion for the selection of the number of clusters and the cluster assignment (see Fig. S5). Details on the clustering implementation and validation are provided in the Supplementary Information.
Codes A set of python codes for running the $S^{3} \mathrm{D}$ analysis are freely available for download on GitHub (https://github.com/ ClementiGroup/S3D).

Acknowledgement We are grateful to D. E. Shaw research for sharing the protein folding simulation data. We are indebted to Peter Koltai (FU Berlin) for inspiring discussions, and to Frank Noé (FU Berlin) for his insightful suggestions and his assistance with the MSM and HMM analysis. This work was supported by the National Science Foundation (CHE-1265929, CHE-1738990, and PHY-1427654), the Welch Foundation (C1570), and the Deutsche Forschungsgemeinschaft through CRC1114. Simulations have been performed on the computer clusters of the Center for Research Computing at Rice University, supported in part by the BigData Private-Cloud Research Cyberinfrastructure MRI-award (NSF grant CNS-1338099), and on the clusters of the Department of Mathematics and Computer Science at Freie Universität, Berlin.

\section{Supporting Information Avail- able}

Details about the model parameters, implementation and validations are provided in the Supporting Information.

\section{References}

(1) Shirts, M.; Pande, V. S. Screen Savers of the World Unite! Science 2000, 290, 1903-1904.

(2) Shaw, D. E.; Maragakis, P.; LindorffLarsen, K.; Piana, S.; Dror, R.; Eastwood, M.; Bank, J.; Jumper, J.; Salmon, J.; Shan, Y.; Wriggers, W. Atomic-Level Characterization of the Structural Dynamics of Proteins. Science 2010, 330, 341-346.

(3) Doerr, S.; Harvey, M. J.; Noé, F.; Fabritiis, G. D. HTMD: High-Throughput 
Molecular Dynamics for Molecular Discovery. J. Chem. Theory Comput. 2016, 12, 1845-1852.

(4) Bowman, G. R., Pande, V. S., Noé, F., Eds. An Introduction to Markov State Models and Their Application to Long Timescale Molecular Simulation.; Advances in Experimental Medicine and Biology; Springer Heidelberg, 2014; Vol. 797.

(5) Rohrdanz, M. A.; Zheng, W.; Clementi, C. Discovering mountain passes via torchlight: methods for the definition of reaction coordinates and pathways in complex macromolecular reactions. Ann. Rev. Phys. Chem. 2013, 64, 295-316.

(6) Noé, F.; Clementi, C. Collective variables for the study of long-time kinetics from molecular trajectories: Theory and methods. Curr. Opin. Struct. Biol. 2017, 43, 141-147.

(7) Plattner, N.; Doerr, S.; De Fabritiis, G.; Noé, F. Complete protein-protein association kinetics in atomic detail revealed by molecular dynamics simulations and Markov modelling. Nature Chemistry 2017, in press.

(8) Shukla, D.; Meng, Y.; Roux, B.; Pande, V. S. Activation pathway of Src kinase reveals intermediate states as targets for drug design. Nat. Commun. 2014, 5, 3397.

(9) Das, P.; Moll, M.; Stamati, H.; Kavraki, L. E.; Clementi, C. Lowdimensional, free-energy landscapes of protein-folding reactions by nonlinear dimensionality reduction. Proc. Natl. Acad. Sci. U.S.A. 2006, 103, 9885-9890.

(10) Kevrekidis, I. G.; Samaey, G. Equationfree multiscale computation: Algorithms and applications. Ann. Rev. Phys. Chem. 2009, 60, 321-344.

(11) Prinz, J.-H.; Wu, H.; Sarich, M.; Keller, B.; Senne, M.; Held, M.; Chodera, J. D.; Schütte, C.; Noé, F.
Markov models of molecular kinetics: generation and validation. J. Chem. Phys. 2011, 134, 174105.

(12) Rohrdanz, M. A.; Zheng, W.; Maggioni, M.; Clementi, C. Determination of reaction coordinates via locally scaled diffusion map. J. Chem. Phys. 2011, 134, 124116.

(13) Schütte, C.; Fischer, A.; Huisinga, W.; Deuflhard, P. A Direct Approach to Conformational Dynamics Based on Hybrid Monte Carlo. J. Comput. Phys. 1999, 151, $146-168$.

(14) Nadler, B.; Lafon, S.; Coifman, R. R.; Kevrekidis, I. G. Adv. Neural Inf. Process. Syst. Adv. Neural Inf. Process. Syst. (NIPS). 2005; pp 955-962.

(15) Clementi, C. Coarse-grained models of protein folding: toy models or predictive tools? Curr. Opin. Struct. Biol. 2008, 18, $10-15$.

(16) Saunders, M. G.; Voth, G. A. Coarsegraining methods for computational biology. Ann. Rev. Biophys. 2013, 42, 73-93.

(17) Noid, W. Perspective: Coarse-grained models for biomolecular systems. $J$. Chem. Phys. 2013, 139, 09B201_1.

(18) Clementi, C.; Nymeyer, H.; Onuchic, J. N. Topological and energetic factors: what determines the structural details of the transition state ensemble and "en-route" intermediates for protein folding? Investigation for small globular proteins. J. Mol. Biol. 2000, 298, 937 - 953.

(19) Davtyan, A.; Schafer, N. P.; Zheng, W.; Clementi, C.; Wolynes, P. G.; Papoian, G. A. AWSEM-MD: Protein structure prediction using coarse-grained physical potentials and bioinformatically based local structure biasing. J. Phys. Chem. B 2012, 116, 8494-8503.

(20) Matysiak, S.; Clementi, C. Minimalist protein model as a diagnostic tool for 
misfolding and aggregation. J. Mol. Biol. 2006, 363, 297-308.

(21) Voth, G. A. Coarse-graining of condensed phase and biomolecular systems; CRC press, 2008.

(22) Potoyan, D. A.; Savelyev, A.; Papoian, G. A. Recent successes in coarsegrained modeling of DNA. Wiley Interdiscip Rev. Comput. Mol. Sci. 2013, 3, 6983.

(23) Buchete, N.-V.; Hummer, G. Coarse master equations for peptide folding dynamics. J. Chem. Phys. B 2008, 112, 60576069 .

(24) Chodera, J. D.; Singhal, N.; Pande, V. S.; Dill, K. A.; Swope, W. C. Automatic discovery of metastable states for the construction of Markov models of macromolecular conformational dynamics. $J$. Chem. Phys. 2007, 126, 04B616.

(25) Noé, F.; Wu, H.; Prinz, J. H.; Plattner, N. Projected and hidden Markov models for calculating kinetics and metastable states of complex molecules. J. Chem. Phys. 2013, 139.

(26) Lindorff-Larsen, K.; Piana, S.; Dror, R. O.; Shaw, D. E. How FastFolding Proteins Fold. Science 2011, 334, $517-520$.

(27) Panchenko, A. R.; Luthey-Schulten, Z.; Wolynes, P. G. Foldons, protein structural modules, and exons. Proc. Natl. Acad. Sci. U. S. A. 1996, 93, 2008-2013.

(28) Ozkan, S. B.; Wu, G. A.; Chodera, J. D.; Dill, K. A. Protein folding by zipping and assembly. Proc. Natl. Acad. Sci. U. S. A. 2007, 104, 11987-11992.

(29) Boninsegna, L.; Gobbo, G.; Noe', F.; Clementi, C. Investigating Molecular Kinetics by Variationally Optimized Diffusion Maps. J. Chem. Theory Comput. 2015, 11, 5947-5960.
(30) Schwantes, C. R.; Pande, V. S. Improvements in Markov State Model Construction Reveal Many Non-Native Interactions in the Folding of NTL9. J. Chem. Theory Comput. 2013, 9, 2000-2009.

(31) Banisch, R.; Koltai, P. Understanding the geometry of transport: Diffusion maps for Lagrangian trajectory data unravel coherent sets. Chaos 2017, 27, 035804.

(32) Froyland, G.; Lloyd, S.; Santitissadeekorn, N. Coherent sets for nonautonomous dynamical systems. Physica $D$ 2010, 239, 1527-1541.

(33) Coifman, R. R.; Lafon, S. Diffusion maps. Appl. Comput. Harm. Anal. 2006, 21, 530.

(34) Zheng, W.; Qi, B.; Rohrdanz, M. A.; Caflisch, A.; Dinner, A. R.; Clementi, C. Delineation of folding pathways of a $\beta$ sheet miniprotein. J. Phys. Chem. B 2011, 115, 13065-13074.

(35) Zheng, W.; Rohrdanz, M. A.; Maggioni, M.; Clementi, C. Polymer reversal rate calculated via locally scaled diffusion map. J. Chem. Phys. 2011, 134, 144109.

(36) Nedialkova, L. V.; Amat, M. A.; Kevrekidis, I. G.; Hummer, G. Diffusion maps, clustering and fuzzy Markov modeling in peptide folding transitions. J. Chem. Phys. 2014, 141, 09B611_1.

(37) Ferguson, A. L.; Panagiotopoulos, A. Z.; Kevrekidis, I. G.; Debenedetti, P. G. Nonlinear dimensionality reduction in molecular simulation: The diffusion map approach. Chem. Phys. Lett. 2011, 509, 111.

(38) Beauchamp, K. a.; McGibbon, R.; Lin, Y.S.; Pande, V. S. Simple few-state models reveal hidden complexity in protein folding. Proc. Natl. Acad. Sci. U.S.A. 2012, 109, 17807-17813.

(39) Hinsen, K. Analysis of domain motions by approximate normal mode calculations. 
Proteins: Struct., Funct., Genet. 1998, 33, 417-429.

(40) Ponzoni, L.; Polles, G.; Carnevale, V.; Micheletti, C. SPECTRUS: A Dimensionality Reduction Approach for Identifying Dynamical Domains in Protein Complexes from Limited Structural Datasets. Structure 2015, 23, 1516 - 1525.

(41) Yesylevskyy, S. O.; Kharkyanen, V. N.; Demchenko, A. P. Dynamic protein domains: identification, interdependence, and stability. Biophys. J. 2006, 91, 670685 .

(42) Sinitskiy, A. V.; Saunders, M. G.; Voth, G. A. Optimal number of coarsegrained sites in different components of large biomolecular complexes. J. Chem. Phys. B 2012, 116, 8363-8374.

(43) Noé, F.; Clementi, C. Kinetic distance and kinetic maps from molecular dynamics simulation. J. Chem. Theory Comput. 2015, 22, 5002-5011.

(44) Noé, F.; Banisch, R.; Clementi, C. Commute maps: separating slowly-mixing molecular configurations for kinetic modeling. J. Chem. Theory Comput. 2016, 12, 5620-5630.

(45) Lange, O. F.; Grubmüller, H. Generalized correlation for biomolecular dynamics. Proteins: Struct., Funct., Bioinf. 2006, 62, 1053-1061.

(46) Dama, J. F.; Sinitskiy, A. V.; McCullagh, M.; Weare, J.; Roux, B.; Dinner, A. R.; Voth, G. A. The theory of ultra-coarse-graining. 1. General principles. J. Chem. Theory Comput. 2013, 9, $2466-2480$.

(47) Gō, M. Correlation of DNA exonic regions with protein structural units in haemoglobin. Nature 1981, 291, 90-92.

(48) Lindberg, M. O.; Oliveberg, M. Malleability of protein folding pathways: a simple reason for complex behaviour. Curr. Opin. Struct. Biol. 2007, 17, 21-29.
(49) Maity, H.; Maity, M.; Krishna, M. M.; Mayne, L.; Englander, S. W. Protein folding: the stepwise assembly of foldon units. Proc. Natl. Acad. Sci. U.S.A. 2005, 102, 4741-4746.

(50) Bryngelson, J. D.; Onuchic, J. N.; Socci, N. D.; Wolynes, P. G. Funnels, pathways, and the energy landscape of protein folding: A synthesis. Proteins: Struct., Funct., Bioinf. 1995, 21, 167195.

(51) Scherer, M. K.; Trendelkamp-Schroer, B.; Paul, F.; Pérez-Hernández, G.; Hoffmann, M.; Plattner, N.; Wehmeyer, C.; Prinz, J.-H.; Noé, F. PyEMMA 2: A Software Package for Estimation, Validation, and Analysis of Markov Models. J. Chem. Theory Comput. 2015, 11, 5525-5542. 
Graphical TOC Entry

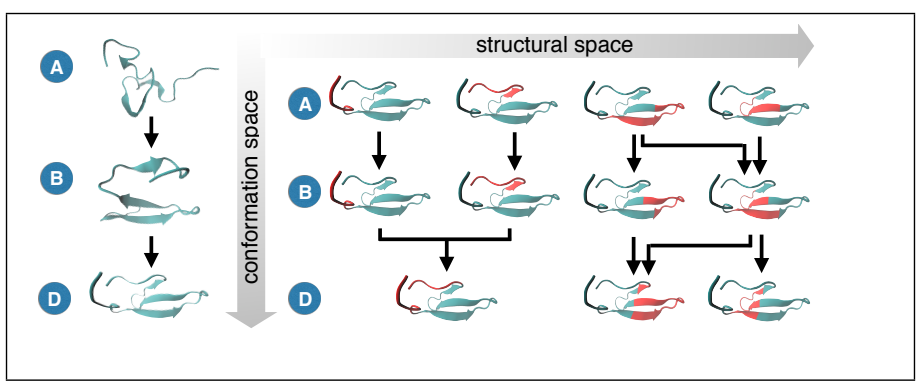




\title{
Supporting information for:
}

\section{A data-driven perspective on the hierarchical assembly of molecular structures}

\author{
Lorenzo Boninsegna, ${ }^{\dagger}$ Ralf Banisch, ${ }^{\ddagger}$ and Cecilia Clementi*,,$\ddagger$ \\ $\dagger$ Department of Chemistry, and Center for Theoretical Biological Physics, Rice University, \\ 6100 Main St, Houston TX 7ro05, USA \\ $\ddagger$ Department of Mathematics and Computer Science, Freie Universität Berlin, Arnimallee \\ 6, 14195 Berlin, Germany \\ E-mail: cecilia@rice.edu
}

\section{Molecular simulation}

Details on the force-field and computational protocol used to generate the all-atom molecular dynamics trajectories for the two proteins considered here (FIP35 WW-domain and NTL9 protein) can be found in the original references. ${ }^{\mathrm{S} 1, \mathrm{~S} 2}$ FIP35 is a 35 residue protein. Two independent $100 \mu \mathrm{s}$ all-atom trajectories in explicit solvent at $337 \mathrm{~K}$ are available. ${ }^{\text {S1 }}$ NTL9 is the 39 residue N-terminal of the ribosomal protein L9. ${ }^{\text {S3 }}$ Four independent all-atom trajectories in explicit solvent at $355 \mathrm{~K}$ are available with lengths of $1052,990,389,377 \mu$ s. $^{\mathrm{S} 2}$

\section{Identification of metastable states}

A Markov State Model analysis of the molecular trajectories was used to extract the metastable states. In short, the analysis consisted of the following steps: 
- A dimensionality reduction of the data was first performed by using the Time-lagged Independent Component Analysis (TICA) ${ }^{\mathrm{S} 4-\mathrm{S} 6}$ and rescaling the resulting reduced coordinates to obtain a commute map ${ }^{\mathrm{S} 7}$ metric space.

- Standard $k$-means clustering was performed in the commute map space to obtain an initial partitioning of the space into a discrete, yet still large $(k=1000)$ number of states (i.e., microstates).

- A maximum likelihood MSM was estimated in the discrete space of $k=1000$ clusters. The final number of metastable states was determined by the presence of a gap in the MSM eigenspectrum, and corresponds to 4 states for both of the proteins considered here (see Fig. S2).

- A Hidden Markov Model (HMM) with the selected number of 4 metastable states was estimated from the discretized commute map space, following a well-established protocol. ${ }^{\mathrm{S} 8}$

Details on the different steps are provided below.

\section{Time-lagged Independent Component Analysis (TICA)}

TICA was used in order to obtain a subspace of reduced dimension while preserving the slow modes of the dynamics. TICA represents a special case of the variational approach for conformational dynamics. ${ }^{\text {S4,S9 }}$

For completeness, we briefly report here the essence of the TICA algorithm (see ${ }^{\mathrm{S} 4, \mathrm{~S} 5}$ for details). The input information is the time evolution of a large set of "features", that is, variables able to trace the slow kinetics, e.g., inter-atomic or inter-residue distances, torsions, and Cartesian coordinates in some reference frame, arbitrary nonlinear functions of molecular coordinates, or any combination of the above. We denote the features as $x_{t, i}$, where $t=1, \ldots, T$, is the time variable (for a trajectory length $\mathrm{T}$ ) and $i=1, \ldots, n$ is an 
index over all the $n$ features. We assume that the features $x_{t, i}$ are mean-free in the time dimension. We define the two matrices:

$$
\mathbf{X}_{0}=\left(\begin{array}{ccc}
x_{1,1} & \ldots & x_{1, n} \\
\vdots & & \vdots \\
x_{T-\tau, 1} & \ldots & x_{T-\tau, n}
\end{array}\right) \quad \mathbf{X}_{\tau}=\left(\begin{array}{ccc}
x_{\tau, 1} & \ldots & x_{\tau, n} \\
\vdots & & \vdots \\
x_{T, 1} & \ldots & x_{T, n}
\end{array}\right)
$$

and compute the correlation matrices:

$$
\begin{aligned}
& \mathbf{C}_{00}=\mathbf{X}_{0}^{T} \mathbf{X}_{0} \\
& \mathbf{C}_{0 \tau}=\mathbf{X}_{\tau}^{T} \mathbf{X}_{0} .
\end{aligned}
$$

We then solve the generalized eigenvalue problem:

$$
\mathbf{C}_{0 \tau} \mathbf{R}=\mathbf{C}_{00} \mathbf{R} \Lambda
$$

where $\mathbf{R}$ is the eigenvector matrix, and $\boldsymbol{\Lambda}$ is the diagonal matrix containing the eigenvalues in descending order. Let $\mathbf{R}^{(m)}$ be the matrix containing the first $m$ rows of $\mathbf{R}$ (that is, the $m$ dominant eigenvectors). The trajectory $\mathbf{X}_{0}$ mapped to the first $m$ TICA coordinates is then given by the transformation:

$$
\boldsymbol{\Psi}_{0}=\mathbf{X}_{0} \mathbf{R}^{(m)}
$$

It can be shown that the TICA coordinates are uncorrelated and have maximal autocovariances at lagtime $\tau .{ }^{\mathrm{S} 5}$ Following the commute map approach introduced in, ${ }^{\mathrm{S} 7}$ the first $m=50$ TICA coordinates were scaled using the square root of the corresponding timescale:

$$
\tilde{\Psi}_{i}=\sqrt{\frac{t_{i}}{t_{0}}} \Psi_{i}
$$

The rescaled TICA coordinates (S3) were used to perform a 1000 microstate clustering of 
the simulation data with $k$-means clustering: As a result, each frame in the configuration space is assigned to a microstate. We indicate the trajectory projected in the commute map space $\left(\boldsymbol{\Psi}_{0}=\mathbf{X}_{0} \mathbf{R}^{(m)}\right.$ from Eq. S2) as $\left\{s_{t}\right\}$.

\section{TICA parameters}

The features selected for the TICA calculation are:

- all closest-heavy atom distances between all residue pairs,

- the reciprocal of all these distances,

- all backbone $(\phi, \psi)$ and side-chain dihedral angles $\left(\chi_{1}, \chi_{2}, \chi_{3}, \chi_{4}, \omega\right)$,

- all dihedral angles defined by the position of any four $C_{\alpha}$ atoms.

The implied timescales resulting from the eigenvalue matrix $\Lambda$ in (S1) for different lag times $\tau$ are shown in Fig. S1. Lag times of $0.04 \mu$ and $0.1 \mu s$ were chosen for FIP35 and NTL9, respectively.
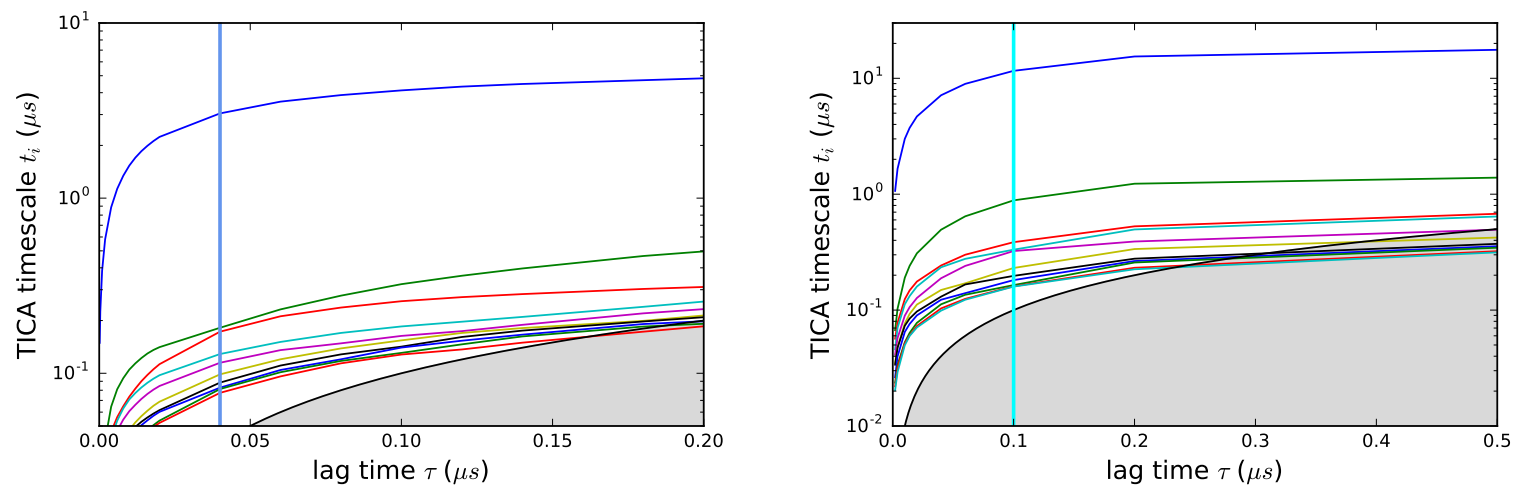

Figure S1: Convergence of TICA implied timescales $t_{i}$ as a function of lag time $\tau$ for FIP35 (left) and NTL9 (right). The vertical line indicates the reference lag time $\tau_{T I C A}$ chosen to define the scaled TICA eigenvectors $\tilde{\Psi}_{i}=\sqrt{t_{i} / t_{0}} \Psi_{i}$ for the clustering step (see text). The grey area represents $t<\tau$; i.e., the unphysical part of the $(t, \tau)$ space. 


\section{Hidden Markov Models}

A coarse grained kinetic model was extracted upon building a Hidden Markov Model ${ }^{\mathrm{S} 8}$ of the discretized trajectories. A Hidden Markov Model (HMM) consists of a transition matrix $\tilde{\mathbf{T}}(\tau)$ between $K$ hidden macrostates (which are here the metastable states we want to identify) with associated stationary distribution $\tilde{\pi}$. The number $K$ of hidden states is a parameter that must be independently estimated, for instance from the existence of a gap in the TICA or MSM eigenspectrum. We indicate by $\chi \in \mathbb{R}^{M \times K}$ the membership matrix, that is each hidden state $k$ has a probability $\chi_{k, m}$ to be in state $m$ of the $N$ available microstates. Microstates are here the large (1000) set of clusters obtained by performing $k$-means clustering on the commute map space (defined by the rescaled TICA coordinates). The observed trajectory is the one projected in the commute map space, $\left\{s_{t}\right\}$. We indicate as $\left\{h_{t}\right\}$ the trajectory projected in the space of the $K$ hidden macrostates we want to identify. We seek to estimate a HMM that allows us to decompose the correlation matrix $\boldsymbol{\Psi}_{\tau}^{T} \boldsymbol{\Psi}_{0}$ between the observable states as:

$$
\boldsymbol{\Psi}_{\tau}^{T} \boldsymbol{\Psi}_{0}=\chi^{T} \tilde{\boldsymbol{\Pi}} \tilde{\mathbf{T}}(\tau) \chi=\chi^{T} \tilde{\mathbf{L}}^{T} \tilde{\boldsymbol{\Lambda}} \tilde{\mathbf{L}} \chi
$$

where the diagonal matrix $\tilde{\Lambda}$ contains the eigenvalues $\tilde{\lambda}_{i}(\tau)$ of the hidden transition matrix $\tilde{\mathbf{T}}(\tau)$.

The likelihood of an HMM defined by $\tilde{\mathbf{T}}(\tau)$ and $\chi \in \mathbb{R}^{M \times K}$ associated with an observed trajectory $\left\{s_{t}\right\}$ (obtained by TICA) is given by: ${ }^{\mathrm{S} 8}$

$$
\mathbb{P}\left(\left\{s_{t}\right\} \mid \tilde{\mathbf{T}}, \chi\right)=\sum_{h_{0}, \ldots, h_{t} \max } \tilde{\pi}_{h_{0}} \chi_{s_{0} h_{0}} \prod_{t=1}^{t_{\max }} \tilde{T}_{h_{t-1} h_{t}} \chi_{s_{t} h_{t}}
$$

The HMM with maximum likelihood is selected.

\section{HMM estimation}

A maximum likelihood Markov State $\mathrm{Model}^{\mathrm{S} 10}$ was first built to infer the appropriate number of states for the coarse grained kinetic model. Fig. S2 shows the MSM implied timescales 
and clearly indicates that either a 2 state or 4 state model is appropriate.
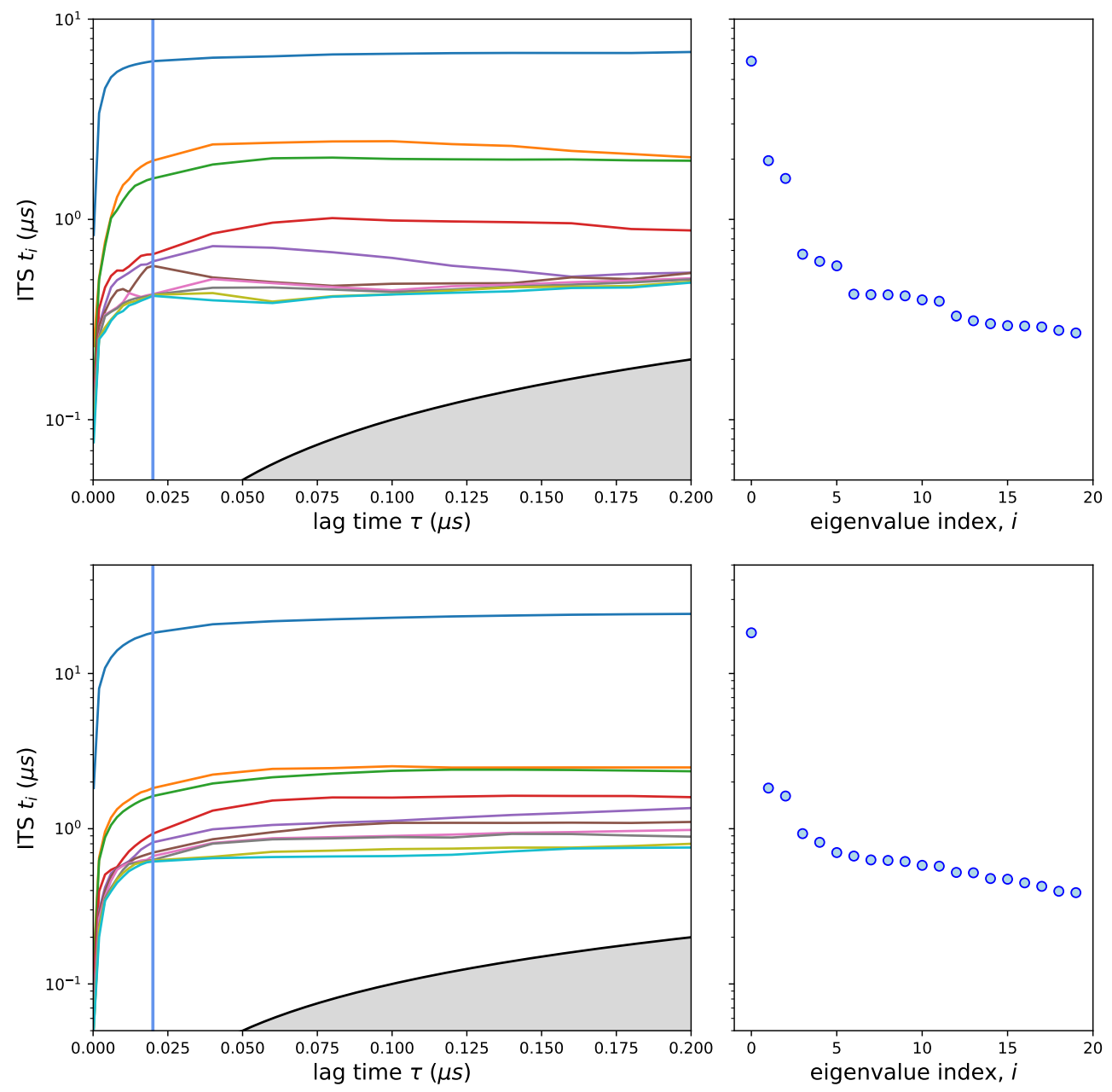

Figure S2: MSM implied time scales for FIP35 (top) and NTL9 (bottom). A thick vertical line indicates an appropriate value of $\tau$ where timescales have converged, and the slice $\left\{t_{k}(\tau)\right\}_{k}$ is plotted in the right panel. Clearly, there are two timescale gaps between $t_{1}$ and $t_{2}$, or between $t_{3}$ and $t_{4}$, suggesting that a 2 or 4 state kinetic coarse grained representation (respectively) is optimal.

As a 2 state model oversimplifies the dynamical features of the macromolecular dynamics by only accounting for direct transitions between the folded and unfolded state, a higher resolution model of $K=4$ was selected for both proteins.

The HMM likelihood (Eq. S4) optimization can be challenging due to the non-convexity of the problem and the combinatorial explosion of the number of hidden paths that can lead to $\left\{s_{t}\right\}$. We use the Baum-Welch expectation maximization algorithm, ${ }^{\text {S11,S12 }}$ together with 
an MSM as an initial guess ${ }^{\mathrm{S} 8}$ for the likelihood optimization. The implementation available in the pyEMMA software ${ }^{\mathrm{S} 13}$ was used.

Because of its probabilistic nature, HMM provides a fuzzy assignment of each molecular configuration into a metastable state. However, a suitable probability threshold can be introduced to obtain a crisp membership matrix, $\bar{\chi}$ as: $\bar{\chi}=\Theta(\chi-\delta)$, where $\Theta$ is the Heavyside function

$$
\Theta(x)= \begin{cases}1, & x \geq 0 \\ 0 & x<0\end{cases}
$$

and we set the threshold value $\delta=0.95$. Only a few trajectory frames did not satisfy this 95\% thresholding criterion and were not assigned to any HMM macrostate, and were then not considered in the analysis that follows. Approximately, only $5 \%$ and $3 \%$ of the total frames available were discarded for FIP35 and NTL9 respectively.

As a result, the high dimensional conformation space visited by the molecule is mapped into a discrete dynamical model consisting of $K$ (here 4) metastable regions, their relative probabilities, and the probability of transition from each state to the others.

\section{HMM validation}

The HMM eigenvalues $\tilde{\lambda}_{i}(\tau)$ of $\tilde{\mathbf{T}}(\tau)$ define the implied timescales:

$$
\tilde{t}_{i}(\tau)=-\frac{\tau}{\log \left|\tilde{\lambda}_{i}(\tau)\right|} .
$$

The convergence of the implied timescales $\tilde{t}_{i}(\tau)$ as a function of lag time $\tau$ is usually used for model validation. ${ }^{\mathrm{S} 8}$ If the $\tilde{t}_{i}(\tau)$ are approximately constant over a broad range of values for $\tau$, then $\tilde{\mathbf{T}}(\tau)$ is approximately Markovian. ${ }^{\mathrm{S} 8}$ In the limit of good statistics, the estimated HMM timescales $\tilde{t}_{i}(\tau)$ are guaranteed to be upper bounded by the true timescales $t_{i}$. 4 ,S14 For these reasons, when considering two models with i.e. different numbers of hidden states $K$ and $K^{\prime}$, the model with larger and more converged timescales is usually chosen. 
For both proteins, we selected a lag time $\tau_{0}$ for the HMM in the converged region where the implied timescales $\tilde{t}_{i}(\tau)$ are approximately constant as a function of $\tau$ (see Figs. S3 and S4). Extensive analysis of $K=2,3,4$ and 5 Hidden Markov Models confirm that $K=2$ or $K=4$ are optimal choices for the number of macrostates, in agreement with the MSM analysis.

Additional validation of the resulting HMM was performed, as follows. First, the hidden states from the membership matrix $\bar{\chi}$ were used to define core sets and the trajectory was mapped to a 4-state core MSM, ${ }^{\mathrm{S} 15}$ following the procedure detailed in. ${ }^{\mathrm{S} 16, \mathrm{~S} 17}$ Then, a four state MSM is built on the core sets and both timescale convergence and the ChapmanKolmogorov (CK) test ${ }^{\mathrm{S} 10}$ were used for validation. Results are shown in fig. S3 and S4 for FIP35 and NTL9 respectively, and indicate that the resulting core set MSMs are stable and satisfy the CK test within the error-bars.

\section{Diffusion Map}

We briefly summarize here the ideas and implementation of the Diffusion Map method for the analysis of molecular simulation. ${ }^{\text {S18-S20 }}$ Let $z_{i, t} \in \mathbb{R}^{3}$ denote the position of atom $i$ at simulation time $t$. We define an $N \times N$ similarity matrix $W_{t}$ with entries:

$$
W_{t, i j}=h\left(\frac{\left\|\hat{z}_{i, t}-\hat{z}_{j, t}\right\|^{2}}{\epsilon}\right),
$$

where $\|\cdot\|$ is a suitable distance, $\epsilon>0$ is a scale parameter, and $h: \mathbb{R} \rightarrow \mathbb{R}_{+}$is a kernel function which is most commonly chosen as Gaussian $h(x)=\exp (-x) 1_{x \leq R}$. A suitably chosen cutoff $R$ sparsifies $\mathbf{W}_{t}$ and ensures only relatively local distances enter the construction. The definion of a distance metric is clearly essential in Diffusion Map to quantify the idea of close or far. As we are looking at three dimensional protein structures in $\mathbb{R}^{3}$, the Euclidean distance $d_{t}(i, j)$ between atoms $i$ and $j$ at time frame $t$ represents a good choice of metric. The Euclidean distance is an unreliable similarity measure in the high dimensional confor- 

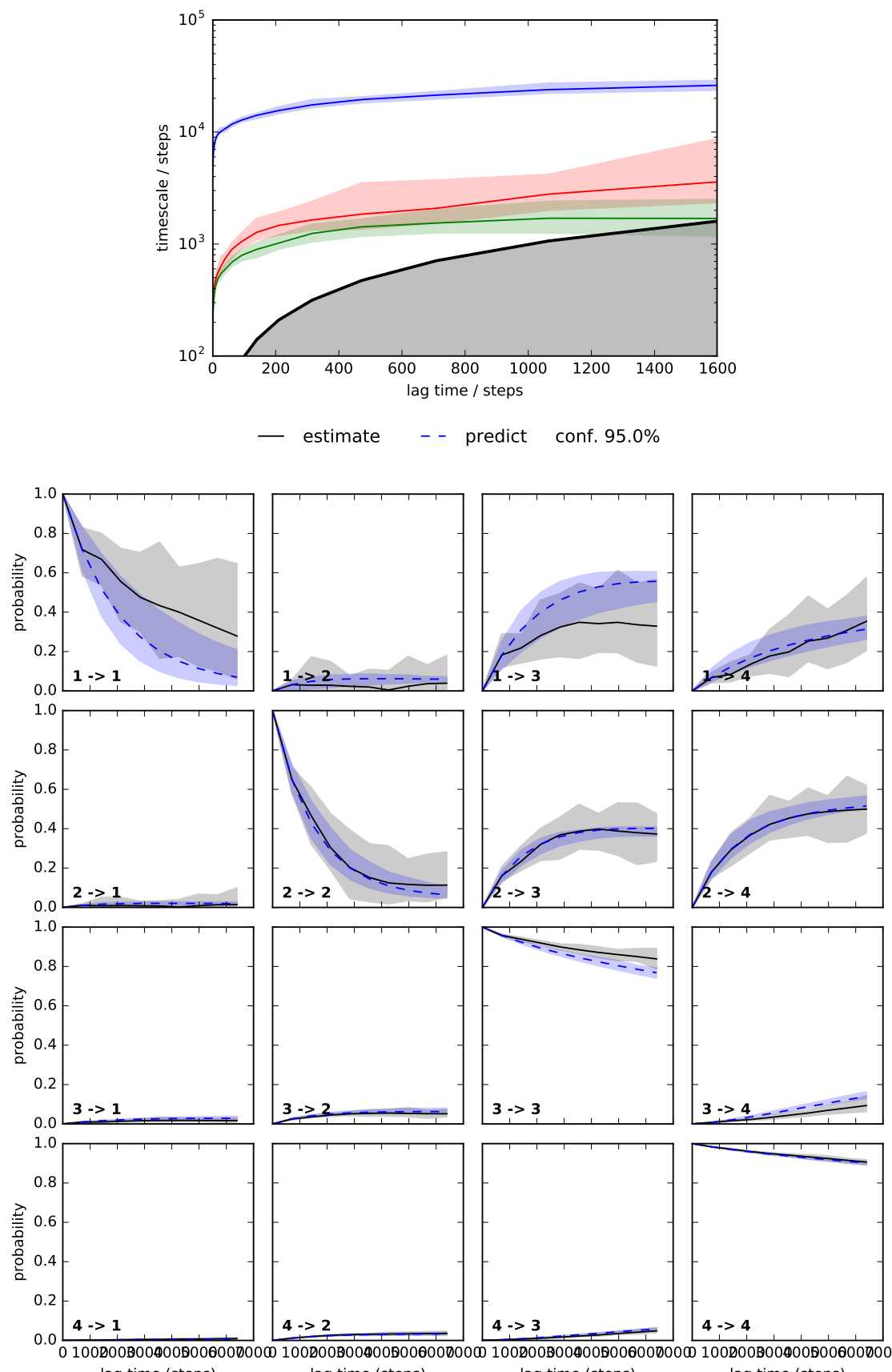

lag time (steps)

lag time (steps)

lag time (steps)

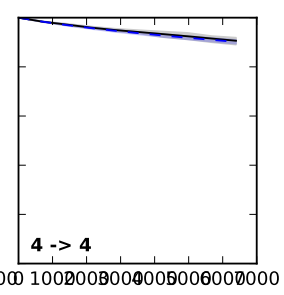

Figure S3: HMM validation for FIP35: core set MSM timescale convergence (top panel) and Chapman-Kolmogorov (CK) test (bottom panel), both with Bayesian error bars. The reference core set MSM for $\mathrm{CK}$ validation was selected at $\tau=0.14 \mu \mathrm{s}=710$ time steps, where convergence is robust. The CK test is positive as there is a good overlap (within the error bars) between the prediction (blue) and the direct estimation (black and grey). 


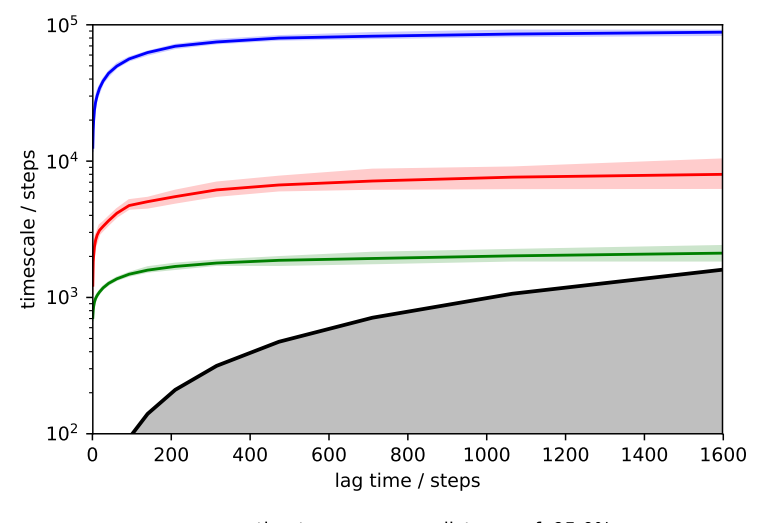

- estimate _- - predict conf. $95.0 \%$
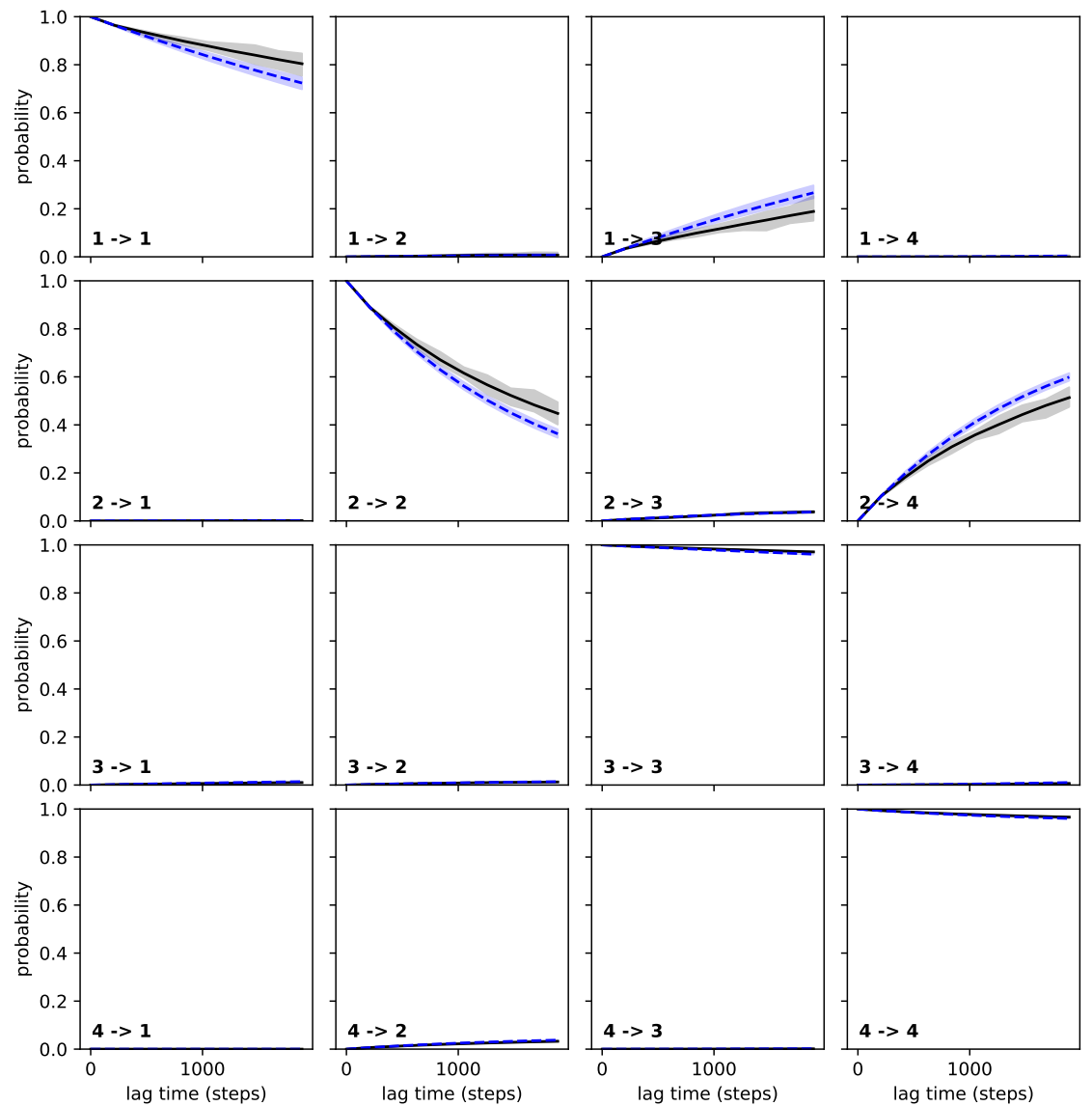

Figure S4: HMM validation for NTL9: core set MSM timescale convergence (top panel) and CK test (bottom panel), both with Bayesian error bars. The reference core set MSM for CK validation was selected at $\tau=0.1 \mu s=500$ time steps, where convergence is robust. As in Fig. S3, the significant overlap (within the error bars) between the prediction (blue) and the direct estimation (black and grey) indicates a positive CK test. 
mation space of a macromolecule, e.g. it is not invariant under translations and rotations of the molecule. This is not a problem in the three dimensional space of a molecule, as considered here. With $\mathbf{D}_{t}$ being the diagonal matrix containing the row sums of $\mathbf{W}_{t}$, the similarity matrix is then normalized to give $\tilde{\mathbf{W}}_{t}=\mathbf{D}_{t}^{-1 / 2} \mathbf{W}_{t} \mathbf{D}_{t}^{-1 / 2}$. Finally, the stochastic matrix $\mathbf{P}_{t}=\tilde{\mathbf{D}}_{t}^{-1} \tilde{\mathbf{W}}_{t}$ is constructed, where $\tilde{\mathbf{D}}_{t}$ is the diagonal matrix containing the row sums of $\tilde{\mathbf{W}}_{t}$, and $\mathbf{P}_{t}-\mathbf{I}$ (where $\mathbf{I}$ is the identity matrix) is a weighted graph Laplacian. It can be shown that the eigenfunctions of $\mathbf{P}_{t}-\mathbf{I}$, or equivalently the eigenfunctions of $\mathbf{P}_{t}$ itself, approximate the eigenfunctions of the Laplace-Beltrami operator which is compatible with the intrinsic geometry of the atom positions $z_{i, t} \in \mathbb{R}^{3}$.

\section{Time-averaged Diffusion Map}

The Structure and State Space Decomposition, $\mathrm{S}^{3} \mathrm{D}$, consists in the identification, for each macrostate $k$, of the groups of atoms that move coherently over time, whenever the macromolecule resides in macrostate $k$. As discussed in the manuscript, this goal is accomplished by using the time-averaged Diffusion Map proposed in. ${ }^{\mathrm{S} 21}$ The ideas and implementation are briefly summarized below.

For a set $I \subset\left\{1, \ldots, T_{I}\right\}$ of time slices, we define the matrix

$$
\mathbf{Q}^{I}=\frac{1}{|I|} \sum_{t \in I} \mathbf{P}_{t}
$$

The matrix $\mathbf{Q}^{I}$ is row stochastic since all the component matrices $\mathbf{P}_{t}$ are, and the entries $Q_{i j}^{I}$ describe the average "closeness" of atoms $i$ and $j$, where the average is over all the time frames considered in $I$. Furthermore, it can be shown that $\mathrm{Q}^{I}$ is $\mathcal{O}\left(\epsilon^{2}\right)$-close to a symmetric matrix. This ensures that the dominant part of the spectrum of $\mathbf{Q}^{I}$ is real.

We diagonalize $\mathbf{Q}^{I}$ :

$$
\mathbf{Q}^{I} \mathbf{U}^{I}=\Lambda^{I} \mathbf{U}^{I}
$$

where $\mathbf{U}^{I}=\left[\mathbf{u}_{1}, \ldots \mathbf{u}_{N}\right]$ is the matrix of eigenvectors and $\boldsymbol{\Lambda}^{I}=\operatorname{diag}\left(\lambda_{1}^{(I)}, \ldots, \lambda_{N}^{(I)}\right)$ contains 
the eigenvalues of $\mathbf{Q}^{I}$. The spectrum of $\mathbf{Q}^{I}$ then contains information about the number of coherent groups, while the dominant eigenvectors $\mathbf{u}_{i}$ encode their location (see below).

This statement can be understood by considering the generator matrix:

$$
\mathbf{L}^{I}=\epsilon^{-1}\left(\mathbf{Q}^{I}-\mathbf{I}\right)
$$

where $\mathbf{I}$ is the $N \times N$ identity matrix, and $\epsilon$ is the scale parameter in (S6). Note that $\mathbf{Q}^{I}$ and $\mathbf{L}^{I}$ have the same eigenvectors. It is possible to show ${ }^{\mathrm{S} 21}$ that $\mathbf{L}^{I}$ is a finite dimensional approximation of the operator

$$
\mathcal{L}^{I}=\frac{1}{|I|} \sum_{t \in I} \mathcal{P}_{t}^{*} \Delta \mathcal{P}_{t}
$$

where $\Delta$ is the Laplacian and $\mathcal{P}_{t}$ is the propagator of the ensemble dynamics weighted by the stationary distribution $\pi$. That is, given an initial distribution of states $\rho_{0}, \mathcal{P}_{t}$ maps this distribution forward to $\rho_{t}$ :

$$
\rho_{t}(y)=\mathcal{P}_{t} \rho_{0}(y)=\frac{1}{\pi(y)} \int p_{t}(x, y) \rho_{0}(x) \pi(x) d x
$$

where the conditional transition probability $p_{t}(x, y)$ governs the dynamics. The approximation of $\mathbf{L}^{I}$ to $\mathcal{L}^{I}$ in $(\mathrm{S} 9)$ is exact in the limit of infinite data and $\epsilon \rightarrow 0$. It was shown ${ }^{\mathrm{S} 21}$ that the dominant eigenfunctions of $\mathcal{L}^{I}$, which are approximated by the $\mathbf{u}_{i}$ 's, encode the locations of dynamically coherent sets. These coherent sets can therefore be uncovered by spectral clustering using $\mathbf{U}^{I}$.

\section{Clusters analysis and validation}

For each protein, a time-averaged Diffusion Map matrix $\mathbf{Q}^{\mathbf{I}}$ according to (S7) was constructed for each of the 4 macrostates identified by the HMM. Then the eigenvalue problem (S8) was solved to obtain the eigenvalues $\boldsymbol{\Lambda}^{I}$ and eigenvectors $\mathbf{U}^{I}$.

Two parameters need to be set in the time-averaged Diffusion Map algorithm: the scale 
parameter $\epsilon>0$, which enters the kernel construction (Eq. S6), and the number of clusters, $n$. Both are chosen by means of the validation method detailed below.

Agglomerative clustering ${ }^{\mathrm{S} 22}$ was used on the space spanned by the first $n$ eigenvectors of the time-average Diffusion Map matrix $\mathbf{Q}^{I}$ to partition the protein atoms into $n$ (coherent) domains. The advantage of hierarchical clustering over other clustering methods (such as the popular $k$-means) is the fact that is a bottom-up approach: each observation (here, each heavy atom) initially starts in a cluster of its own, and pairs of clusters are progressively merged as more iterations are run, i.e. as one moves up the hierarchy (technically, up the dendrogram, see Fig. S5(d) and (h)). The algorithm relies on an intrinsic distance threshold (Ward distance) $\mathcal{D}$ associated with each iteration (i.e. with a given number of clusters created $n_{i}$ ), that is the distance between the last two clusters that have been aggregated; i.e. an upper bound for the dissimilarity of the clusters. Such distance threshold can be monitored as a function of the number of clusters $\mathcal{D}_{i}=\mathcal{D}\left(n_{i}\right)$ : if this distance is very small more clusters than optimal are defined. On the other hand, a sudden increase in this distance indicates that two clusters quite dissimilar from each other have been merged, that is, less clusters than optimal are being used. Thus, a gap in $\mathcal{D}_{i}$ as a function of $n_{i}$ signals that an appropriate number of iterations have been performed to assign the data to the a suitable number of clusters.

A commonly used heuristic criterion for choosing an optimal number of clusters in spectral clustering consists in looking for gaps in the eigenvalue spectrum, since such a gap $\cdots \lambda_{i-1}>$

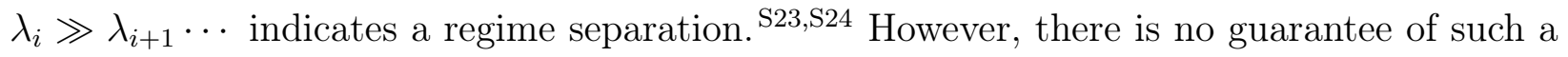
scenario and most of the times the eigenvalue spectrum is a continuum. Therefore we use a more robust cluster validation, based on the idea of the silhouette score, which measures how similar a datum is to its own cluster compared to other clusters. ${ }^{\text {S25-S27 }}$ For each heavy atom $i$ in the protein under consideration, we compute

$$
\mathcal{S}(i)=\frac{\mathcal{B}(i)-\mathcal{A}(i)}{\max (\mathcal{A}(i), \mathcal{B}(i))}
$$


Here $\mathcal{A}(i)$ is the average similarity (or dissimilarity) of $i$ with all other data within the same cluster, and $\mathcal{B}(i)$ is the lowest average dissimilarity of $i$ to any other cluster of which $i$ is not a member. The Ward distance of the agglomerative clustering is used as a distance metric to compute $\mathcal{A}(i)$ and $\mathcal{B}(i)$. Definition S10 implies that $-1 \leq \mathcal{S}(i) \leq 1, \forall i$, specifically:

- $\mathcal{S}(i) \rightarrow 1$ indicates that $\mathcal{A}(i) \ll \mathcal{B}(i)$; hence, $i$ is well-clustered

- $\mathcal{S}(i) \approx 1$ indicates that $\mathcal{A}(i) \approx \mathcal{B}(i)$; hence, $i$ could be equally assigned to another cluster (probably too few clusters are used).

- $\mathcal{S}(i) \rightarrow-1$ indicates that $\mathcal{A}(i) \gg \mathcal{B}(i)$; hence, $i$ is probably mis-assigned to its cluster.

The average (or the median) $\mathcal{\mathcal { S }}$ score over all dataset is a good measure of how appropriately the data have been clustered.

A representative example of the validation procedure is presented in figure S5 for the NTL9 folded state, where the results are illustrated both an "optimal" $n=3$ and "non optimal" $n=5$ choice for the number of clusters (left and right panels respectively). Panels (b) and (c) show that $n=3$ is associated with a clear drop in the Ward distance threshold (see the gap identified by the vertical line in panel (b)). Consistently, the silhouette score (panel (c)) is large up to $n_{i}=3$ clusters, and drops when fewer clusters (i.e. more clustering iterations) are used. The results are robust on a broad range of scale parameters $\epsilon$. In contrast, panels (f) and (g) show that $n=5$ clusters would be a bad clustering choice for the data, as no clear signal is returned in the validation: neither a large drop in the distance threshold (panel (f)) nor a significant change in the silhouette score (panel (g)). It is worth noting that using the position of a possible spectral gap in the eigenvalue plot $-\epsilon / \log \lambda_{k}$ (Fig. S5, panels (a) and (e)) is not as conclusive as the validation test provided by computing the distance threshold and silhouette score. The dendrograms associated with both clustering choices are also displayed in panels (d) and (h) for a given value of the scale parameter ( $\epsilon=0.3 \mathrm{~nm}$, which is the value selected for all the subsequent analysis). Horizontal dashed black lines highlight the gap $\mathcal{D}_{n_{k} \rightarrow n_{k}-1}^{n}$ in the Ward distance as the chosen number of clusters 
(a)

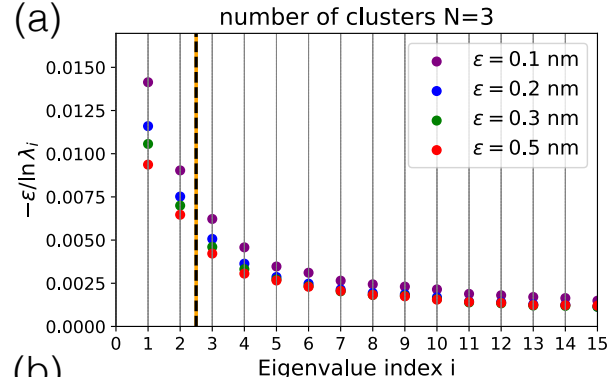

(b)

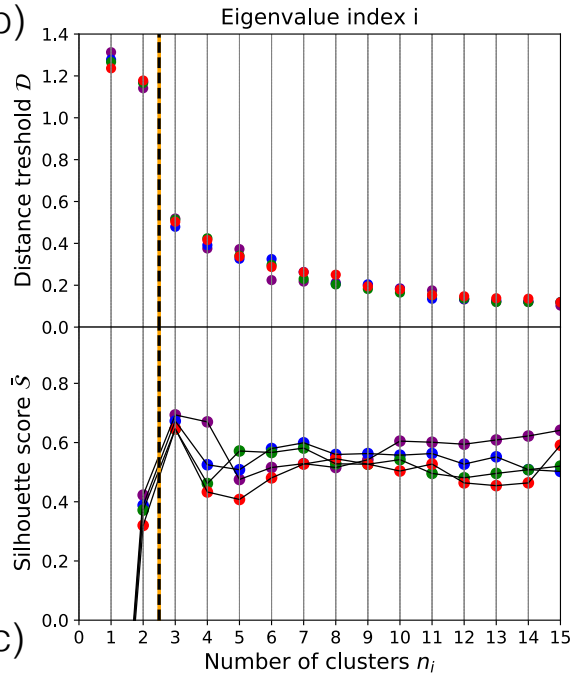

(d)

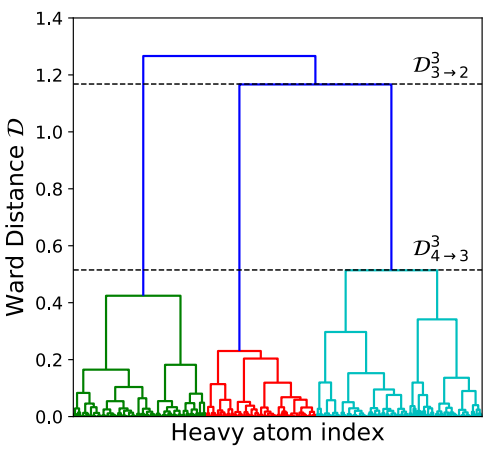

number of clusters $\mathrm{N}=5$

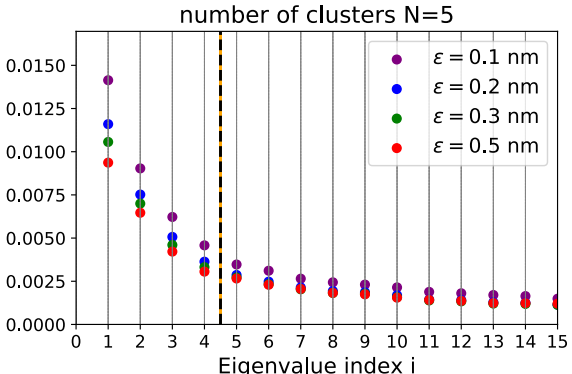

$(e)$

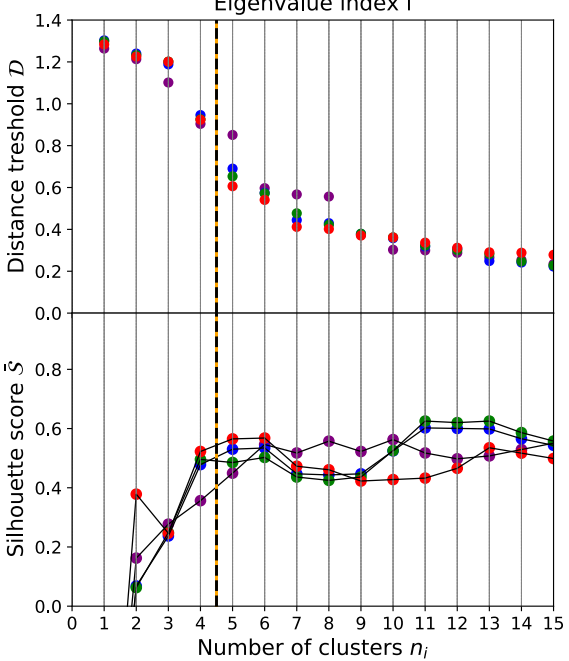

(f)

(g)

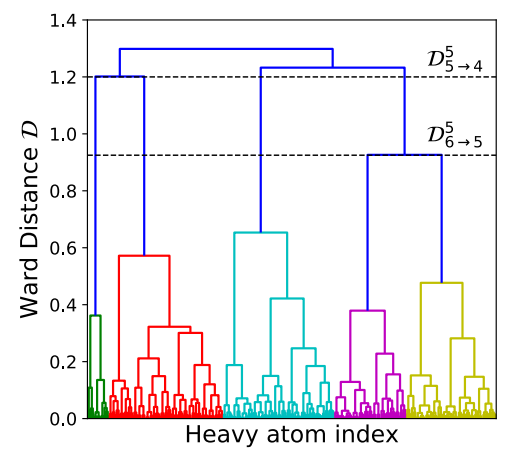

(h)

Figure S5: Cluster validation procedure applied to the folded state of NTL9. Panels on the left (a)-(d) (and (e)-(h) on the right) summarize the main results for an optimal (nonoptimal) choice of the number of clusters $n, n=3$ and $n=5$ clusters respectively. The following quantities are displayed: a "normalized" eigenvalue spectrum $-\epsilon / \log \lambda_{k}$ (panels (a) and (e)), the Ward distance threshold $\mathcal{D}$ (panels (b) and (f)), and the average silhouette score $\overline{\mathcal{S}}$ (panels (c) and (g)), as a function of the clustering iteration/number of clusters $n_{i}$. All these quantities are plotted for different values of the scale parameter $\epsilon$. A thick vertical line is drawn in correspondence of the number of clusters corresponding to the number of eigenvectors used in the clustering, i.e., at $n_{i}=n$. In addition, the dendrograms associated with the agglomerative clustering are displayed for the value of scale parameter $\epsilon=0.3 \mathrm{~nm}$ (panels (d) and (h)). Dashed thin horizontal lines in the dendrograms indicate the critical Ward distance values which mark the number of clusters $n_{i}=n$ (compare with panels (b) and (f)). Specifically, $\mathcal{D}_{n_{k} \rightarrow n_{k}-1}^{n}$ describes the threshold distance associated with the iteration reducing the number of clusters from $n_{k}$ to $n_{k}-1$. 
$n=n_{i}$ is reached. As already shown in panels (b) and (f), a substantial distance gap exists for $n=3$, whereas it is much smaller for $n=5$.

All clustering calculations were performed using the implementation available in the scikit-learn package (scikit-learn.org). ${ }^{\text {S28 }}$ A python code for running the cluster validation analysis as detailed above is freely available for download on GitHub (https://github. com/ClementiGroup/S3D).

\section{Cluster decomposition for the less populated folding pathways}

The results from the application of the $\mathrm{S}^{3} \mathrm{D}$ to both proteins are shown in the main text for the metastable states populated along the most likely folding pathways (see Fig. 1). In Fig. S6 we show the results along the less likely pathways. As in Fig. 1, a schematic is given on the left representing the HMM kinetic network, while the results from the $\mathrm{S}^{3} \mathrm{D}$ are shown on the right. As in Fig. 1, the splitting and merging of structural domains regulates the dynamics of the two proteins. A more detailed analysis is provided in the main text.

\section{Foldons calculation}

Foldons were extracted from the all-atom trajectories following the procedure detailed in. ${ }^{\mathrm{S} 29}$ Structural quantities such as the fractions of native $Q$, and of non-native contacts $A$, and the radius of gyration $R_{g}$ were computed for each frame and used to define an ensemble of folded and misfolded structures, with the cutoff values $\left(C_{q+}, C_{r g}, C_{q-}, C_{a q}\right)$ listed in Table S1:

$$
\text { folded }=\left\{\begin{array}{l}
Q \geq C_{q+} \\
R_{g} \leq C_{r g}
\end{array} \quad \text { misfolded }=\left\{\begin{array}{l}
Q \leq C_{q-} \\
A+Q \geq C_{a q} \\
R_{g} \leq C_{r g}
\end{array}\right.\right.
$$

Following, ${ }^{\text {S29 }}$ the quantity $\Theta=\frac{\Delta E}{\sqrt{N} \delta E}$ is a measure of local foldability of protein segments, where $\Delta E$ is the stability gap, i.e. the energy difference between the folded structure and the ensemble of compact misfolded structures, $\delta E$ is the energy variance of of the misfolded 

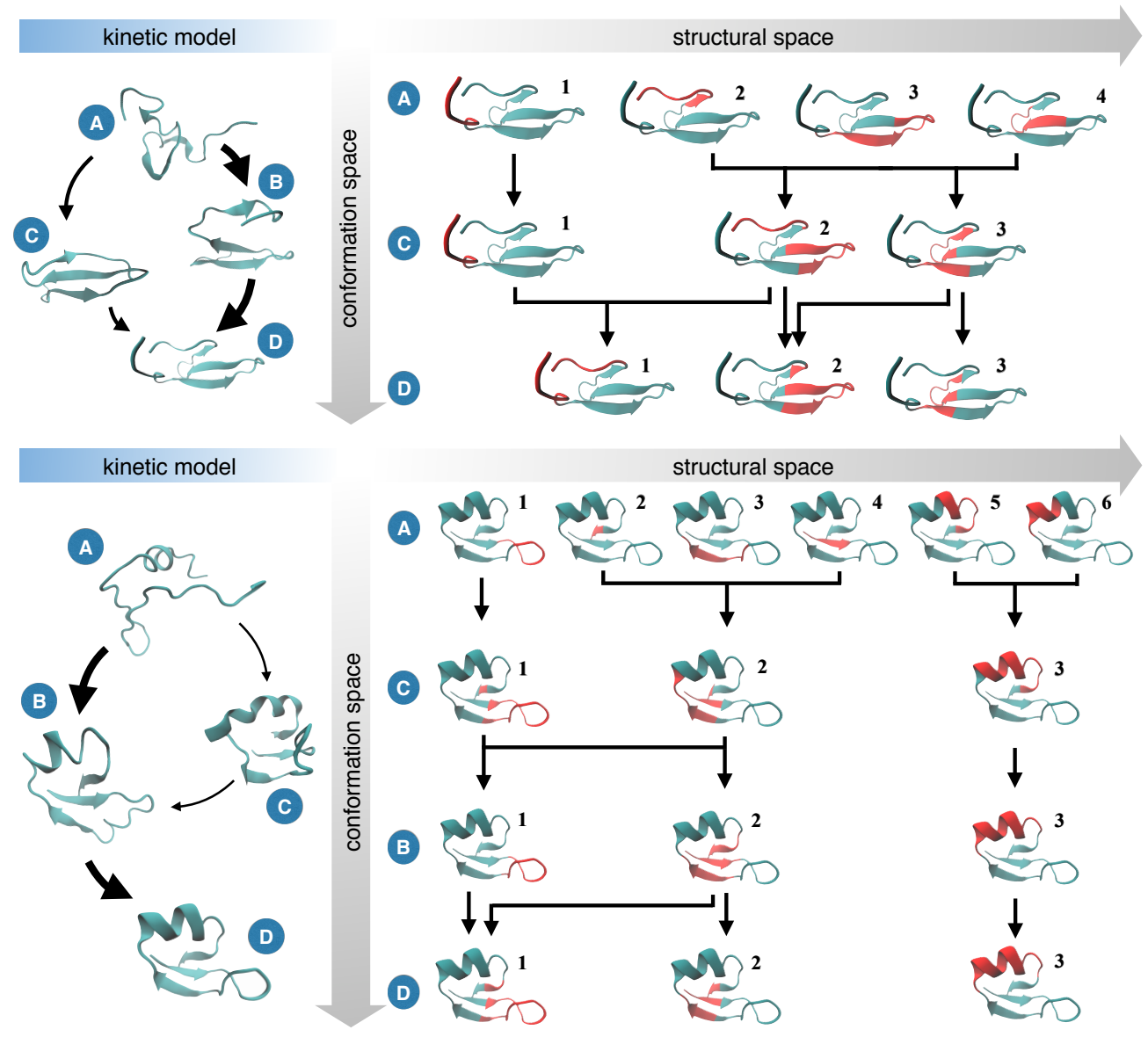

Figure S6: Kinetic network and $\mathrm{S}^{3} \mathrm{D}$ results for FIP35 (upper panel) and NTL9 (lower panel) proteins. (Left panels) A schematic representation of the Hidden Markov Model network; transition fluxes are indicated with arrows of widths proportional to the values of the transition probabilities. (Right panels) Results from the $\mathrm{S}^{3} \mathrm{D}$ for the metastable states involved in the less likely folding pathway $(A \rightarrow C \rightarrow D$ and $A \rightarrow C \rightarrow B \rightarrow D$ for FIP35 and NTL9, respectively). Structural domains within a given state are labeled with numbers and are colored in red on the folded structure to facilitate comparison between different states. Black arrows connecting the domains are used as visual aid to show how the clusters identity changes as the system transitions from the unfolded $(A)$ to the folded state $(D)$. 
Table S1: List of parameters used to assign configurations either to the folded or misfolded ensemble.

\begin{tabular}{ccccc}
\hline \hline protein & $C_{q+}$ & $C_{q-}$ & $C_{a q}$ & $C_{r g}$ \\
\hline FIP35 & 0.65 & 0.40 & 0.75 & 0.75 \\
NTL9 & 0.75 & 0.50 & 0.75 & 0.75 \\
\hline
\end{tabular}

structures, and $N$ the number of residues of the segment.

Computationally, we estimate $\Delta E$ as the energy difference between the peaks of the distributions of folded and misfolded structures, and $\delta E$ as the standard deviation of the misfolded energy distribution.

Foldon boundaries were defined according to the following procedure $\left(\mathrm{see}^{\mathrm{S} 29}\right)$. The protein chain $\mathcal{P}$ was cleaved after a given residue $j$ along the sequence, and the average $\Theta_{j}$ of the two segments $\mathcal{P}_{[N, j]}$ (N-terminus) and $\mathcal{P}_{(j, C]}$ (C-terminus) was computed $\Theta_{j}=\frac{\Theta_{[N, j]}+\Theta_{(j, C]}}{2}$. The cleavage point was progressively moved along the chain and the calculation repeated. Local maxima in the $\Theta_{j}$ as a function of the cleavage position $j$ are indicators of foldon boundaries, see Fig. S7.

After the first foldon is located $\mathcal{F}_{[N, \bar{j}]}$, the cleaving procedure is replicated from the very beginning on the complementary segment $\mathcal{P}_{(\bar{j}+1, C]}$, the first maximum along this iteration indicating the rightmost boundary of the second foldon (the leftmost being residue $\bar{j}+1$ ), and so on.

In order to extract the parameters $\left\{\Delta E_{j}, \delta E_{j}\right\}$ for the computation of the local foldabilities, energy minimization was performed on all protein segments $\mathcal{P}_{[N, j]}, \mathcal{P}_{(j, C]} \forall j$ for each frame in the folded $(f o l)$ and misfolded (mis) structures, with atomistic force field as those used in the original references ${ }^{\text {S1,S2 }}$ (AMBER99 FS-ILDN for FIP35 ${ }^{\text {S30 }}$ and CHARM22* for NTL9 ${ }^{\mathrm{S} 31}$ ), but using implicit solvent instead of explicit water molecules. As implicit solvent, a Generalized Born approximation to the exact Poisson-Boltzmann model was used, augmented with the hydrophobic solvent accessible surface area (GBSA). Specifically, Born radii were calculated using Hawkins-Cramer-Truhlar method (HCT) ${ }^{\mathrm{S} 32}$ and the Analytical Continuum Electrostatics (ACE) ${ }^{\mathrm{S} 33}$ approximation was used to specify non-polar solvation. 

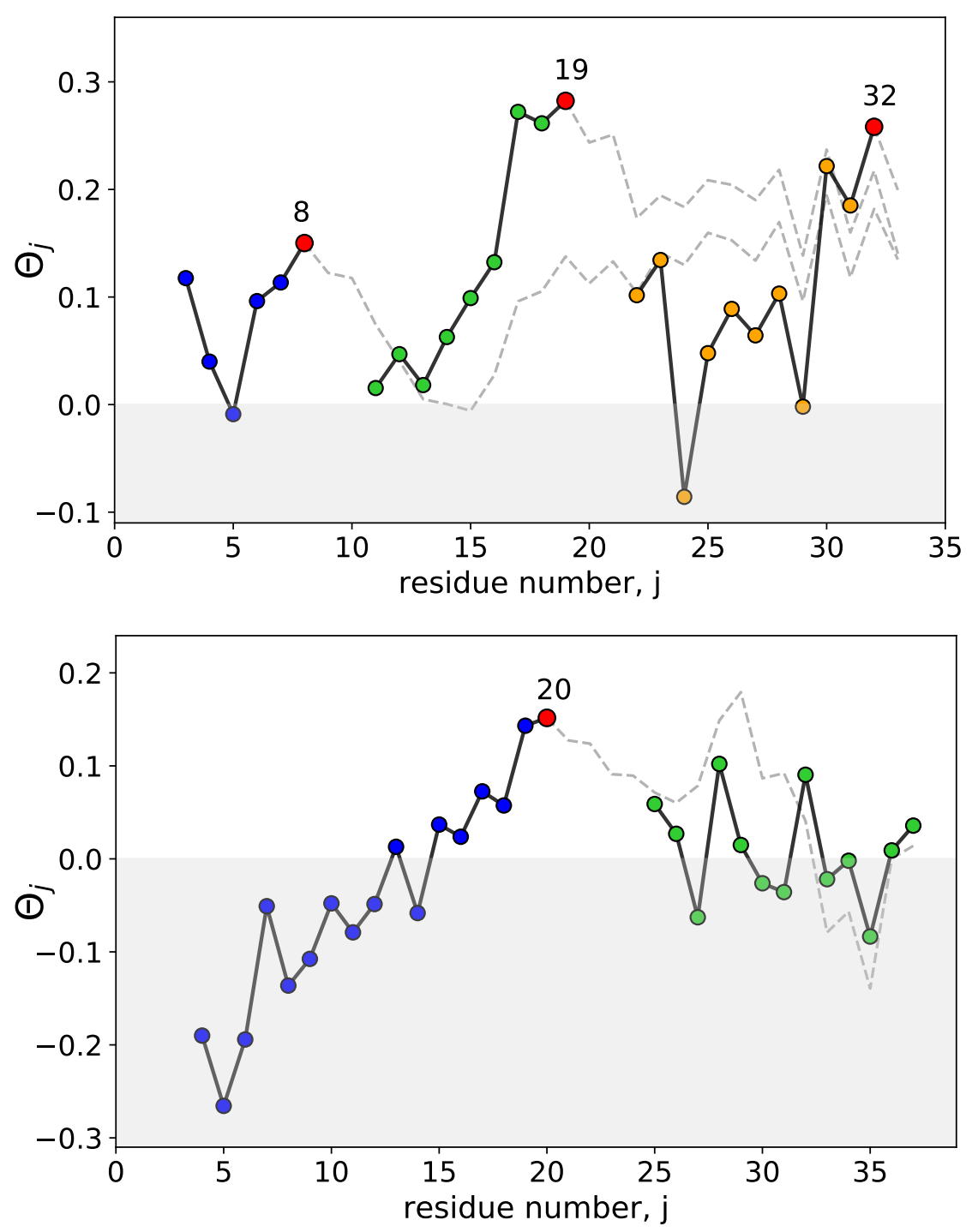

Figure S7: $\Theta_{j}$ as a function of the cleavage position $j$ along the protein chain, for FIP35 (upper panel) and NTL9 (lower panel). Negative values indicate that the corresponding segments do not fold spontaneously as their misfolded structures are lower in energy, on average, than their structures in the native configuration. Red dots indicate the local maxima locating foldon boundaries (hence the point where the cleavage procedure is restarted), and different colors are used to indicate the results of different iterations, whereas dashed lines show the results of the cleaving iteration beyond the foldon boundary, for completeness. FIP35 is decomposed into four foldons, while NTL9 is decomposed into two foldons.

Energy minimization calculations were performed using the GROMACS software. ${ }^{\text {S34 }}$

For each protein segment, histograms were build and parameters determined as follows:

$$
\Delta E_{j}=\left\langle E_{m i s}^{j}\right\rangle-\left\langle E_{f o l}^{j}\right\rangle, \quad \delta E_{j}=\sigma\left(E_{m i s}^{j}\right)
$$


Fig. S7 suggests that the following foldon decompositions are appropriate:

$$
\mathcal{P}_{\text {FIP35 }}=[1,8] \cup[9,19] \cup[20,32] \cup[33,35] \quad \mathcal{P}_{\text {NTL9 }}=[1,20] \cup[21,39]
$$

A discussion of the foldon results within the framework of the $S^{3} D$ approach and and their implications is given in the main manuscript.

\section{References}

(S1) Shaw, D. E.; Maragakis, P.; Lindorff-Larsen, K.; Piana, S.; Dror, R.; Eastwood, M.; Bank, J.; Jumper, J.; Salmon, J.; Shan, Y.; Wriggers, W. Atomic-Level Characterization of the Structural Dynamics of Proteins. Science 2010, 330, 341-346.

(S2) Lindorff-Larsen, K.; Piana, S.; Dror, R. O.; Shaw, D. E. How Fast-Folding Proteins Fold. Science 2011, 334, 517-520.

(S3) Hoffman, D. W.; Davies, C.; Gerchman, S. E.; Kycia, J. H.; Porter, S. J.; White, S. W.; Ramakrishnan, V. Crystal structure of prokaryotic ribosomal protein L9: a bi-lobed RNA-binding protein. EMBO J. 1994, 13, 205-212.

(S4) Noé, F.; Nüske, F. A variational approach to modeling slow processes in stochastic dynamical systems. Multiscale Model. Simul. 2013, 11, 635-655.

(S5) Pérez-Hernández, G.; Paul, F.; Giorgino, T.; De Fabritiis, G.; Noé, F. Identification of slow molecular order parameters for Markov model construction. J. Chem. Phys. 2013, 139, 015102.

(S6) Schwantes, C. R.; Pande, V. S. Improvements in Markov State Model Construction Reveal Many Non-Native Interactions in the Folding of NTL9. J. Chem. Theory Comput. 2013, 9, 2000-2009. 
(S7) Noé, F.; Banisch, R.; Clementi, C. Commute maps: separating slowly-mixing molecular configurations for kinetic modeling. J. Chem. Theory Comput. 2016, 12, 56205630.

(S8) Noé, F.; Wu, H.; Prinz, J. H.; Plattner, N. Projected and hidden Markov models for calculating kinetics and metastable states of complex molecules. J. Chem. Phys. 2013, 139.

(S9) Nüske, F.; Keller, B. G.; Pérez-Hernández, G.; Mey, A. S. J. S.; Noé, F. Variational Approach to Molecular Kinetics. J. Chem. Theory Comput. 2014, 10, 1739-1752.

(S10) Prinz, J.-H.; Wu, H.; Sarich, M.; Keller, B.; Senne, M.; Held, M.; Chodera, J. D.; Schütte, C.; Noé, F. Markov models of molecular kinetics: generation and validation. J. Chem. Phys. 2011, 134, 174105.

(S11) Baum, L. E.; Petrie, T.; Soules, G.; Weiss, N. A maximization technique occurring in the statistical analysis of probabilistic functions of Markov chains. Ann. Math. Stat. $1970,41,164-171$.

(S12) Welch, L. R. Hidden Markov models and the Baum-Welch algorithm. IEEE Information Theory Society Newsletter 2003, 53, 10-13.

(S13) Scherer, M. K.; Trendelkamp-Schroer, B.; Paul, F.; Pérez-Hernández, G.; Hoffmann, M.; Plattner, N.; Wehmeyer, C.; Prinz, J.-H.; Noé, F. PyEMMA 2: A Software Package for Estimation, Validation, and Analysis of Markov Models. J. Chem. Theory Comput. 2015, 11, 5525-5542.

(S14) Djurdjevac, N.; Sarich, M.; Schütte, C. Estimating the eigenvalue error of Markov state models. Multiscale Model. Simul. 2012, 10, 61-81.

(S15) Vanden-Eijnden, E.; Venturoli, M. Markovian milestoning with Voronoi tessellations. J. Chem. Phys. 2009, 130. 
(S16) Schütte, C.; Noé, F.; Lu, J.; Sarich, M.; Vanden-Eijnden, E. Markov state models based on milestoning. J. Chem. Phys. 2011, 134.

(S17) Sarich, M.; Banisch, R.; Hartmann, C.; Schütte, C. Markov state models for rare events in molecular dynamics. Entropy 2014, 16, 258-286.

(S18) Coifman, R. R.; Lafon, S. Diffusion maps. Appl. Comput. Harm. Anal. 2006, 21, 5-30.

(S19) Nadler, B.; Lafon, S.; Coifman, R. R.; Kevrekidis, I. G. Adv. Neural Inf. Process. Syst. Adv. Neural Inf. Process. Syst. (NIPS). 2005; pp 955-962.

(S20) Rohrdanz, M. A.; Zheng, W.; Maggioni, M.; Clementi, C. Determination of reaction coordinates via locally scaled diffusion map. J. Chem. Phys. 2011, 134, 124116.

(S21) Banisch, R.; Koltai, P. Understanding the geometry of transport: Diffusion maps for Lagrangian trajectory data unravel coherent sets. Chaos 2017, 27, 035804.

(S22) Hastie, T.; Tibshirani, R.; Friedman, J. The Elements of Statistical Learning; Springer Series in Statistics; Springer New York Inc.: New York, NY, USA, 2001.

(S23) Ng, A. Y.; Jordan, M. I.; Weiss, Y. On spectral clustering: Analysis and an algorithm. Adv. Neural Inf. Process. Syst. 2002; pp 849-856.

(S24) Von Luxburg, U. A tutorial on spectral clustering. Stat. Comput. 2007, 17, 395-416.

(S25) Rousseeuw, P. J. Silhouettes: A graphical aid to the interpretation and validation of cluster analysis. J. Comput. Appl. Anal. 1987, 20, 53-65.

(S26) Calligari, P.; Gerolin, M.; Abergel, D.; Polimeno, A. Decomposition of Proteins into Dynamic Units from Atomic Cross-Correlation Functions. J. Chem. Theory Comput. 2017, 13, 309-319.

(S27) Nguyen, T.; Habeck, M. A probabilistic model for detecting rigid domains in protein structures. Bioinformatics 2016, 32, i710-i717. 
(S28) Pedregosa, F.; Varoquaux, G.; Gramfort, A.; Michel, V.; Thirion, B.; Grisel, O.; Blondel, M.; Prettenhofer, P.; Weiss, R.; Dubourg, V.; Vanderplas, J.; Passos, A.; Cournapeau, D.; Brucher, M.; Perrot, M.; Duchesnay, E. Scikit-learn: Machine Learning in Python. J. Mach. Learn. Res. 2011, 12, 2825-2830.

(S29) Panchenko, A. R.; Luthey-Schulten, Z.; Wolynes, P. G. Foldons, protein structural modules, and exons. Proc. Natl. Acad. Sci. U. S. A. 1996, 93, 2008-2013.

(S30) Lindorff-Larsen, K.; Piana, S.; Palmo, K.; Maragakis, P.; Klepeis, J. L.; Dror, R. O.; Shaw, D. E. Improved side-chain torsion potentials for the Amber ff99SB protein force field. Proteins: Struct., Funct., Bioinf. 2010, 78, 1950-1958.

(S31) Piana, S.; Lindorff-Larsen, K.; Shaw, D. E. How robust are protein folding simulations with respect to force field parameterization? Biophys. J. 2011, 100, L47-L49.

(S32) Hawkins, G. D.; Cramer, C. J.; Truhlar, D. G. Parametrized models of aqueous free energies of solvation based on pairwise descreening of solute atomic charges from a dielectric medium. J. Phys. Chem. 1996, 100, 19824-19839.

(S33) Schaefer, M.; Karplus, M. A comprehensive analytical treatment of continuum electrostatics. J. Phys. Chem. 1996, 100, 1578-1599.

(S34) Abraham, M. J.; Murtola, T.; Schulz, R.; Páll, S.; Smith, J. C.; Hess, B.; Lindahl, E. GROMACS: High performance molecular simulations through multi-level parallelism from laptops to supercomputers. SoftwareX 2015, 1, 19-25. 